\title{
Loops in AdS: from the spectral representation to position space. Part II
}

\section{Dean Carmi}

Haifa Research Center for Theoretical Physics \& Astrophysics, University of Haifa, 199 Aba Khoushy Ave. Mt. Carmel, Haifa, Israel

E-mail: deancarmi1@gmail.com

Abstract: We continue the study of AdS loop amplitudes in the spectral representation and in position space. We compute the finite coupling 4-point function in position space for the large- $N$ conformal Gross Neveu model on $A d S_{3}$. The resummation of loop bubble diagrams gives a result proportional to a tree-level contact diagram. We show that certain families of fermionic Witten diagrams can be easily computed from their companion scalar diagrams. Thus, many of the results and identities of [1] are extended to the case of external fermions. We derive a spectral representation for ladder diagrams in AdS. Finally, we compute various bulk 2-point correlators, extending the results of [1].

KEYworDS: Conformal Field Theory, Effective Field Theories

ArXiv EPrint: 2104.10500 


\section{Contents}

1 Introduction 1

2 Witten diagrams with external fermions 2

$2.1\langle\bar{\psi} \psi \bar{\psi} \psi\rangle$

$2.2\langle\bar{\psi} \psi \phi \phi\rangle$

2.3 More general fermionic correlators 4

2.4 Spectral representation 5

3 The Gross-Neveu model on $\boldsymbol{A d S}_{3} \quad 6$

4 Ladder diagrams in AdS $\quad 7$

4.1 Warmup: tree-level comb Witten diagrams 8

4.2 4-point ladder diagrams 9

4.32 and 3-point ladder diagrams 11

4.4 Mixed ladders/bubbles 11

$\begin{array}{lll}4.5 & \phi^{4} \text { ladders } & 12\end{array}$

5 2-point bulk correlators in AdS 13

$\begin{array}{ll}\text { A Ladder diagram at 1-loop } & 18\end{array}$

B An eigenvalue equation for ladder diagrams? 20

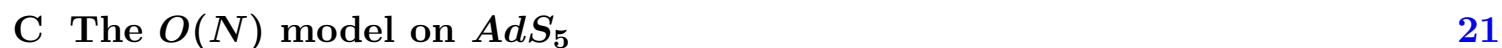

D Scalar 4-point bubble diagrams in $A d S_{5} \quad 24$

E Scalar 4-point bubble diagrams in $A d S_{2} \quad 27$

\section{Introduction}

Two very important problems in physics are: understanding quantum gravity and understanding strongly coupled (non-perturbative) quantum field theories. These two problems are beautifully connected to each other via the gauge-gravity duality, in which Anti-de Sitter (AdS) space plays a unique role. Therefore computing observables for quantum field theories defined on AdS space gives important insights about the two questions mentioned above. AdS space, being a maximally symmetric space-time, often enables to perform analytic computations of observables in the QFT on AdS. For a very partial list of references see $[2-13]$. 

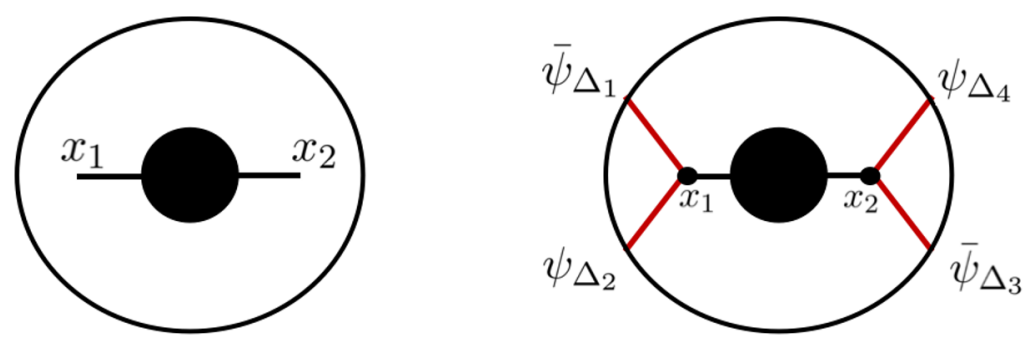

Figure 1. Left: the black blob represents a general bulk two-point corelator $F\left(x_{1}, x_{2}\right)$, with points $x_{1}$ and $x_{2}$. Right: attaching fermionic bulk-to-boundary propagators (red lines) to the 2-point blob gives a CFT 4-point function. This class of diagrams has 2 bulk-to-boundary propagators attached to $x_{1}$ and $x_{2}$, and 2 attached to $x_{2}$.

An important observable for a quantum field theory defined on AdS space is the scattering amplitude. The amplitude can be computed perturbatively, similarly to Feynman diagrams in flat space. Through the AdS/CFT correspondence, AdS scattering amplitudes compute CFT correlation functions on the boundary of AdS. Computations of AdS scattering amplitudes have been performed in position-space [14-23], in Mellin-space [24-31], momentum-space [32-38], embedding space [39, 40]. Most of these computations are restricted to tree-level amplitudes. Other computations rely on the conformal symmetry or the supersymmetry of the dual theory [41-54]. For additional works on AdS amplitudes and in particular loop amplitudes, see [55-71].

In this work we continue the study of loop Witten diagrams initiated in [1], and extend those results in several directions. In section 2 we consider Witten diagrams with external fermions. Using an identity between bulk-to-boundary fermion and scalar propagators, we show that certain families of fermionic diagrams can be directly computed from similar diagrams with external scalars. In section 3 we compute the finite coupling 4-point function of the conformal large- $N$ Gross-Neveu model on $A d S_{3}$. The result of an infinite sum of loop bubble diagrams is extremely simple: it is proportional to a tree-level contact diagram 4-point function. In section 4 we consider scalar ladder diagrams in AdS with an arbitrary number of rungs. We derive a spectral representation for ladder diagrams in AdS. In section 5 we compute bulk-to-bulk 2-point correlators in several cases: scalar sunset diagrams in $A d S_{3}$, and bubble diagrams for scalars in $A d S_{5}$ and for fermions in $A d S_{3}$. In appendix A we show the details of the calculation of the spectral representation of the 1-loop box diagram. In appendix $\mathrm{B}$ we speculate about an eigenvalue equation for ladder diagrams in AdS. In appendix $\mathrm{C}$ we compute a 4-point correlator for the $O(N)$ model on $A d S_{5}$. In appendix D we discuss 4-point bubble diagrams for a scalar $\phi^{4}$ theory on $A d S_{5}$. In appendix E we discuss 4-point bubble diagrams for a scalar $\phi^{4}$ theory on $A d S_{2}$.

\section{Witten diagrams with external fermions}

In [1] we considered scalar Witten diagrams, and derived identities which enabled us to compute various families of loop diagrams in AdS. In the current section we consider 
Witten diagrams containing external fermions. We show that a class of fermionic diagrams is proportional to their scalar companion diagrams.

\section{$2.1\langle\bar{\psi} \psi \bar{\psi} \psi\rangle$}

Consider a (scalar) 2-point bulk correlator $F\left(x_{1}, x_{2}\right)$, which is represented by the black blob in figure 1-Left. We consider the correlator of 4 external fermions $\psi$ with scaling dimensions $\left(\Delta_{1}, \Delta_{2}, \Delta_{3}, \Delta_{4}\right)$, attached to the 2-point blob $F\left(x_{1}, x_{2}\right)$, see figure 1-Right. This 4-point function is:

$$
\left\langle\bar{\psi}_{\Delta_{1}}\left(P_{1}, S_{1}\right) \psi_{\Delta_{2}}\left(P_{2}, S_{2}\right) \bar{\psi}_{\Delta_{3}}\left(P_{3}, S_{3}\right) \psi_{\Delta_{4}}\left(P_{4}, S_{4}\right)\right\rangle
$$

where $P_{i}$ are points on the boundary of $\operatorname{AdS}$ and $S_{i}$ is a spinor polarization variable. For further details see e.g. section 6 of [55]. The fermionic bulk-to-boundary propagators $K_{\Delta}^{F}$ are given by:

$$
\begin{aligned}
K_{\Delta}^{F} \equiv K_{\Delta}^{F}\left(x, \bar{S}_{b}, P, S_{\partial}\right) & =\sqrt{\mathcal{C}_{\Delta}^{F}} \frac{\bar{S}_{b} \Pi_{-} S_{\partial}}{(-2 x \cdot P)^{\Delta+\frac{1}{2}}}, \\
\bar{K}_{\Delta}^{F} \equiv \bar{K}_{\Delta}^{F}\left(x, S_{b}, P, \bar{S}_{\partial}\right) & =\sqrt{\mathcal{C}_{\Delta}^{F}} \frac{\bar{S}_{\partial} \Pi_{-} S_{b}}{(-2 x \cdot P)^{\Delta+\frac{1}{2}}}, \\
\text { where } \quad \mathcal{C}_{\Delta}^{F} & \equiv \frac{1}{\pi^{d / 2}} \frac{\Gamma\left(\Delta+\frac{1}{2}\right)}{\Gamma\left(\Delta+\frac{1-d}{2}\right)},
\end{aligned}
$$

where $x$ is a bulk point, $P$ a boundary point, and $\Delta$ is the scaling dimension of the fermion. Let us define:

$$
S_{12 \mid 34}=\left(\bar{S}_{2 \partial} \Pi_{-} S_{1 \partial}\right)\left(\bar{S}_{4 \partial} \Pi_{-} S_{3 \partial}\right), \quad S_{14 \mid 32}=\left(\bar{S}_{4 \partial} \Pi_{-} S_{1 \partial}\right)\left(\bar{S}_{2 \partial} \Pi_{-} S_{3 \partial}\right) .
$$

where $\Pi_{ \pm}$are chiral projectors in embedding space. The diagram of figure 1-Right is given by:

$$
\begin{aligned}
S_{12 \mid 34} \widehat{g}_{4}^{F}= & \int d^{d+1} x_{1} d^{d+1} x_{2} F\left(x_{1}, x_{2}\right)\left(\partial_{S_{1 b}} \partial_{\bar{S}_{2 b}}\right) \bar{K}_{\Delta_{1}}^{F}\left(x_{1}, S_{1 b}, P_{1}, \bar{S}_{1 \partial}\right) K_{\Delta_{2}}^{F}\left(x_{1}, \bar{S}_{2 b}, P_{2}, S_{2 \partial}\right) \\
& \times\left(\partial_{S_{3 b}} \partial_{\bar{S}_{4 b}}\right) \bar{K}_{\Delta_{3}}^{F}\left(x_{2}, S_{3 b}, P_{3}, \bar{S}_{3 \partial}\right) K_{\Delta_{4}}^{F}\left(x_{2}, \bar{S}_{4 b}, P_{4}, S_{4 \partial}\right)
\end{aligned}
$$

where the integrals $\int d^{d+1} x_{1} d^{d+1} x_{2}$ are over the $A d S_{d+1}$ space. Now we use the following identity: ${ }^{1}$

$$
\begin{aligned}
& \left(\partial_{S_{1 b}} \partial_{\bar{S}_{2 b}}\right) \bar{K}_{\Delta_{1}}^{F}\left(x_{1}, S_{1 b}, P_{1}, \bar{S}_{1 \partial}\right) K_{\Delta_{2}}^{F}\left(x_{1}, \bar{S}_{2 b}, P_{2}, S_{2 \partial}\right) \\
& =\sqrt{\left(2 \Delta_{1}+1-d\right)\left(2 \Delta_{2}+1-d\right)}\left(\bar{S}_{1 \partial} \Pi_{-} S_{2 \partial}\right) K_{\Delta_{1}+\frac{1}{2}}\left(x_{1}, P_{1}\right) K_{\Delta_{2}+\frac{1}{2}}\left(x_{1}, P_{2}\right),
\end{aligned}
$$

where $K_{\Delta}^{F}$ are fermionic bulk-to-boundary fermionic propagators, and $K_{\Delta}$ are scalar bulkto-boundary propagators. This identity enables to write eq. (2.6) in terms of scalar propagators:

$$
\begin{aligned}
S_{12 \mid 34} \widehat{g}_{4}^{F}= & S_{12 \mid 34}\left(\prod_{i=1}^{4} \sqrt{\left(2 \Delta_{i}+1-d\right)}\right) \int d^{d+1} x_{1} d^{d+1} x_{2} F\left(x_{1}, x_{2}\right) \\
& \times K_{\delta_{1}}\left(P_{1}, x_{1}\right) K_{\delta_{2}}\left(P_{2}, x_{1}\right) K_{\delta_{3}}\left(P_{3}, x_{2}\right) K_{\delta_{4}}\left(P_{4}, x_{2}\right)
\end{aligned}
$$

\footnotetext{
${ }^{1}$ See [72], and also eq. 6.19 of [55].
} 

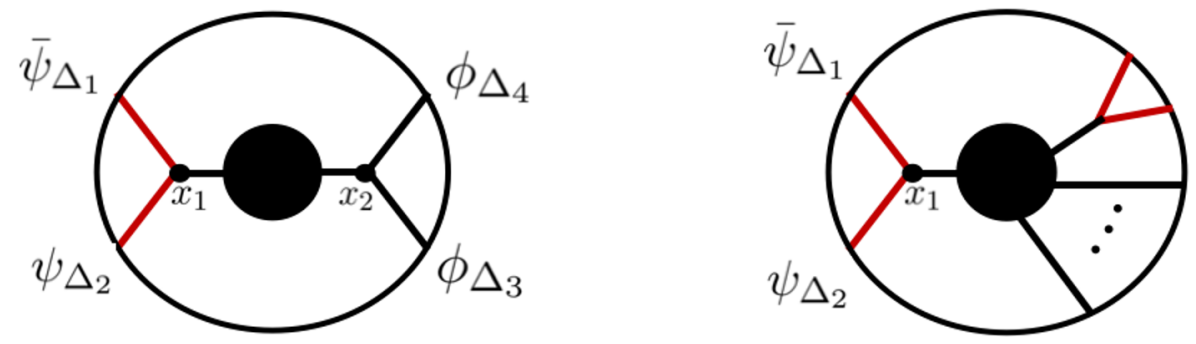

Figure 2. Left: like figure 1-right, only here the $x_{2}$ vertex has scalar propagators attached to it. Right: a completely general diagram in AdS, which has a vertex $x_{1}$ to which are attached two fermionic bulk-to-boundary propagators.

where we defined $\delta_{i}=\Delta_{i}+\frac{1}{2}$. The second line contains a product of 4 scalar bulk-toboundary propagators with dimension $\delta_{i}$. Thus we showed that the fermionic diagram is equal, up to a factor, to the scalar diagram with shifted scaling dimensions $\delta_{i}=\Delta_{i}+\frac{1}{2}$.

Example. Consider the contact tree-level diagram of 4-fermions with scaling dimensions $\Delta_{i}$. Using eq. (2.8) we see that this diagram is proportional to contact diagram of four scalar with scaling dimensions $\delta_{i}=\Delta_{i}+\frac{1}{2}$. It is well known that the scalar contact diagram is computed via special functions called $\bar{D}$ functions, Thus:

$$
\widehat{g}_{4}^{F}(z, \bar{z}) \sim \bar{D}_{\Delta_{1}+\frac{1}{2}, \ldots, \Delta_{4}+\frac{1}{2}}
$$

\section{$2.2\langle\bar{\psi} \psi \phi \phi\rangle$}

Consider the 4-point correlator with 2 external scalars and 2 external fermions, connected to a general 2-point correlator, as in figure 2-Left. We have:

$$
\begin{aligned}
& \int d^{d+1} x_{1}^{d+1} d x_{2} F\left(x_{1}, x_{2}\right) \\
& \times\left(\partial_{S_{1 b}} \partial_{\bar{S}_{2 b}}\right) \bar{K}_{\Delta_{1}}^{F}\left(x_{1}, S_{1 b}, P_{1}, \bar{S}_{1 \partial}\right) K_{\Delta_{2}}^{F}\left(x_{1}, \bar{S}_{2 b}, P_{2}, S_{2 \partial}\right) K_{\Delta_{3}}\left(P_{3}, x_{2}\right) K_{\Delta_{4}}\left(P_{4}, x_{2}\right)
\end{aligned}
$$

where $F\left(x_{1}, x_{2}\right)$ is an arbitrary bulk-to-bulk 2-point function. Using the identity of eq. (2.7) in eq. (2.10), gives:

$$
\begin{aligned}
& \sqrt{\left(2 \Delta_{1}+1-d\right)\left(2 \Delta_{2}+1-d\right)}\left(\bar{S}_{1 \partial} \Pi_{-} S_{2 \partial}\right) \\
& \times \int d^{d+1} x_{1} d^{d+1} x_{2} F\left(x_{1}, x_{2}\right) K_{\Delta_{1}+\frac{1}{2}}\left(x_{1}, P_{1}\right) K_{\Delta_{2}+\frac{1}{2}}\left(x_{1}, P_{2}\right) K_{\Delta_{3}}\left(P_{3}, x_{2}\right) K_{\Delta_{4}}\left(P_{4}, x_{2}\right)
\end{aligned}
$$

which is a 4 -point correlator with 4 external scalars.

\subsection{More general fermionic correlators}

Consider a Witten diagram with 2 external fermions attached to a single vertex, as in figure 2-Right. The rest of the diagram is completely arbitrary. Using the identity of eq. (2.7) gives:

$$
\int d^{d+1} x_{1}\left(\partial_{S_{1 b}} \partial_{\bar{S}_{2 b}}\right) \bar{K}_{\Delta_{1}}^{F}\left(x_{1}, S_{1 b}, P_{1}, \bar{S}_{1 \partial}\right) K_{\Delta_{2}}^{F}\left(x_{1}, \bar{S}_{2 b}, P_{2}, S_{2 \partial}\right) \times(\ldots)
$$


where $(\ldots)$ represents the rest of the diagram, which is arbitrary. Using the identity of eq. (2.7) gives

$$
\begin{aligned}
& \sqrt{\left(2 \Delta_{1}+1-d\right)\left(2 \Delta_{2}+1-d\right)}\left(\bar{S}_{1 \partial} \Pi_{-} S_{2 \partial}\right) \\
& \times \int d^{d+1} x_{1} K_{\Delta_{1}+\frac{1}{2}}\left(x_{1}, P_{1}\right) K_{\Delta_{2}+\frac{1}{2}}\left(x_{1}, P_{2}\right) \times(\ldots)
\end{aligned}
$$

Therefore a vertex containing 2 external fermions can be replaced with a vertex containing 2 external scalars, and the rest of the diagram stays the same. As a result, many of the identities derived in [1] for external scalars, can be applied to diagrams with external fermions.

\subsection{Spectral representation}

In this section we recall the spectral representation of the 4-point correlator of 4 fermions, attached to a 2-point function in the bulk as in figure 1-Right. We denote this correlator by $\widehat{g}_{4}^{F}(z, \bar{z})$, and define the stripped fermionic correlator $g_{4}(z, \bar{z})$ as follows:

$$
g_{4}(z, \bar{z}) \equiv \frac{\widehat{g}_{4}^{F}}{A_{\delta_{i}}^{F}}
$$

where we defined the prefactor: ${ }^{2}$

$$
A_{\delta_{i}}^{F} \equiv c \frac{\frac{\left(\frac{P_{14}^{2}}{P_{24}^{2}}\right)^{\frac{\delta_{2}-\delta_{1}}{2}}\left(\frac{P_{14}^{2}}{P_{13}^{2}}\right)^{\frac{\delta_{3}-\delta_{4}}{2}}}{\sqrt{\Gamma_{\delta_{1}} \Gamma_{\delta_{2}} \Gamma_{\delta_{3}} \Gamma_{\delta_{4}} \Gamma_{\delta_{1}-\frac{\delta_{2}}{2}+1} \Gamma_{\delta_{2}-\frac{d}{2}+1} \Gamma_{\delta_{3}-\frac{d}{2}+1} \Gamma_{\delta_{4}-\frac{d}{2}+1}}}\left(\prod_{i=1}^{4} \sqrt{\left(2 \delta_{i}-d\right)}\right)}{{\frac{\delta}{3}+\delta_{4}}}
$$

where $c$ is a numerical factor which will be unimportant for us.

In eq. (2.8) the fermionic diagram was directly related to that of 4 scalars. Now one can write the spectral representation of this (see eq. 6.20 of [55].). The integrand in eq. (2.8) contains the bulk 2-point function $F\left(x_{1}, x_{2}\right)$, which has a spectral representation:

$$
F\left(x_{1}, x_{2}\right)=\int_{-\infty}^{\infty} d \nu \tilde{F}(\nu) \Omega_{\nu}\left(x_{1}, x_{2}\right)
$$

where the AdS harmonic function $\Omega_{\nu}\left(x_{1}, x_{2}\right)$ is a linear combination of a bulk-to-bulk propagator and it's shadow partner:

$$
\Omega_{\nu}\left(x_{1}, x_{2}\right)=\frac{i \nu}{2 \pi}\left(G_{\frac{d}{2}+i \nu}\left(x_{1}, x_{2}\right)-G_{\frac{d}{2}-i \nu}\left(x_{1}, x_{2}\right)\right)
$$

One can now derive the spectral representation of the 4-point function. The result is:

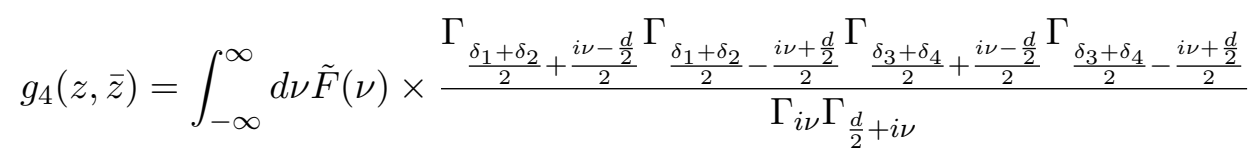

$$
\begin{aligned}
& \times\left(\Gamma_{\frac{\delta_{2}-\delta_{1}}{2}+\frac{i \nu+\frac{d}{2}}{2}} \Gamma_{\frac{\delta_{1}-\delta_{2}}{2}+\frac{i \nu+\frac{d}{2}}{2}} \Gamma_{\frac{\delta_{3}-\delta_{4}}{2}+\frac{i \nu+\frac{d}{2}}{2}} \Gamma_{\frac{\delta_{4}-\delta_{3}}{2}+\frac{i \nu+\frac{d}{2}}{2}}\right) \mathcal{K}_{\frac{d}{2}+i \nu}^{\delta_{i}}(z, \bar{z})
\end{aligned}
$$

\footnotetext{
${ }^{2}$ See eqs. C.10-C.11 of [1].
} 


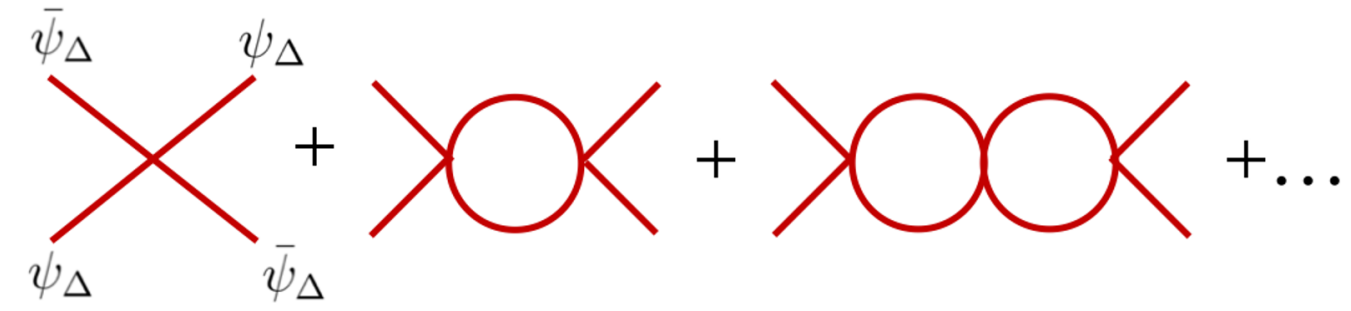

Figure 3. The Gross-Neveu model on AdS. The 4-point correlator at large- $N$ is given by an infinite sum of bubble diagrams. The red lines are fermionic propagators. The boundary of AdS is not shown in the figure.

where $\mathcal{K}_{\frac{d}{2}+i \nu}^{\delta_{i}}(z, \bar{z})$ is the scalar conformal block with scaling dimensions $\delta_{i}=\Delta_{i}+\frac{1}{2}$, and $\tilde{F}(\nu)$ is the spectral representation of the bulk 2-point function $F\left(x_{1}, x_{2}\right)$, eq. (2.16). If in eq. (2.18) one chooses to close the contour in the $\nu$-plane and pick up the residues of the poles, one would get the conformal block expansion of the 4-point correlator.

\section{The Gross-Neveu model on $\boldsymbol{A d S}_{3}$}

Consider the Gross-Neveu [73] model containing $N$ spin-1/2 Dirac fermions $\psi^{i}$, with $i=1, \ldots, N$, see also $[74,75]$. In flat-space and large- $N$ this is a solvable model, exhibiting chiral symmetry breaking and asymptotic freedom. The lagrangian is:

$$
\mathcal{L}=\bar{\psi}^{i} \gamma \cdot \nabla \psi^{i}+m \bar{\psi}^{i} \psi^{i}+\frac{g}{2 N}\left(\bar{\psi}^{i} \psi^{i}\right)^{2}
$$

We denote the scaling dimension of the $\psi$ 's as $\Delta$. In [55] we considered the Gross-Neveu model on $A d S_{d+1}$, and computed the finite coupling 4-point correlator in the spectral representation (This is the same as eq. (2.18) with $\Delta_{i}=\Delta$.):

$$
g_{4}(z, \bar{z})=\int d \nu \tilde{F}(\nu) \frac{\Gamma_{\Delta+\frac{1}{2}-\frac{d+2 i \nu}{4}}^{2} \Gamma_{\Delta+\frac{1}{2}-\frac{d-2 i \nu}{4}}^{2} \Gamma_{\frac{d+2 i \nu}{4}}^{4}}{\Gamma_{i \nu} \Gamma_{\frac{d}{2}+i \nu}} \mathcal{K}_{\frac{d}{2}+i \nu}^{\Delta}(z, \bar{z}),
$$

where the spectral function $\tilde{F}(\nu)$ is a resummation of 1-loop bubble diagrams, as in figure 3 . In this section we compute the $\nu$ integral in eq. (3.2) and thus derive explicitly an expression for the 4-point correlator $g_{4}(z, \bar{z})$ in position-space.

The spectral function $\tilde{F}(\nu)$ was computed [55] explicitly in $d=1,2$. Let us consider the case of $d=2$, i.e $A d S_{3}$, in which the resummation of bubble diagrams gives:

$$
\tilde{F}(\nu)=\frac{1}{\frac{-i\left(4(\Delta-1)^{2}+\nu^{2}\right)}{8 \pi \nu}\left(\psi\left(\Delta+\frac{i \nu}{2}\right)-\psi\left(\Delta-\frac{i \nu}{2}\right)\right)+\frac{2 \Delta-1}{8 \pi(\Delta-1)}}
$$

where the digamma function is $\psi(x)=d \log \Gamma(x) / d x$, not to be confused with the notation for the fermion $\psi$. [55] showed evidence for a bulk conformal point when the external scaling dimension is $\Delta=3 / 2$. Plugging $\Delta=3 / 2$ in eq. (3.3), the spectral function simplifies:

$$
\tilde{F}(\nu)=\frac{8 \nu}{\left(1+\nu^{2}\right) \tanh \left(\frac{\pi \nu}{2}\right)}=\frac{8(h-1)}{h(h-2) \cot \left(\frac{\pi h}{2}\right)}
$$


where we defined $h \equiv \frac{d}{2}+i \nu$. Now we use an identity for the conformal block (see eq. 3.5 of $[1]$.):

$$
2 D_{z, \bar{z}} \mathcal{K}_{\frac{d}{2}+i \nu}(z, \bar{z})=-\left(\nu^{2}+\frac{d^{2}}{4}\right) \mathcal{K}_{\frac{d}{2}+i \nu}(z, \bar{z})
$$

where the operator $D_{z, \bar{z}} \equiv z^{2}(1-z) \partial_{z}^{2}-z^{2} \partial_{z}+\bar{z}^{2}(1-\bar{z}) \partial_{\bar{z}}^{2}-\bar{z}^{2} \partial_{\bar{z}}$ for $d=2$. The scalar conformal block in $d=2$ can be written in terms of LegendreQ functions:

$$
\mathcal{K}_{\beta}(z, \bar{z})=k_{\beta}(z) k_{\beta}(\bar{z})=4 \frac{\Gamma^{2}(\beta)}{\Gamma^{4}\left(\frac{\beta}{2}\right)} Q_{\frac{\beta}{2}-1}(\hat{z}) Q_{\frac{\beta}{2}-1}(\hat{\bar{z}})
$$

where $k_{\beta}(z) \equiv z^{\frac{\beta}{2}}{ }_{2} F_{1}\left(\frac{\beta}{2}+a, \frac{\beta}{2}+b, \beta, z\right), a \equiv \frac{\Delta_{21}}{2}=0$ and $b \equiv \frac{\Delta_{34}}{2}=0$, and we defined:

$$
\hat{z} \equiv \frac{2}{z}-1, \quad \hat{\bar{z}} \equiv \frac{2}{\bar{z}}-1
$$

Combining eqs. (3.2)-(3.6), gives:

$$
g_{4}(z, \bar{z})=D_{z, \bar{z}} \int d \nu \frac{-8 \pi^{2} \nu^{2}}{\sinh (\pi \nu)} Q_{\frac{i \nu-1}{2}}(\hat{z}) Q_{\frac{i \nu-1}{2}}(\hat{\bar{z}})
$$

Now we close the contour in the $\nu$-plane and use the residue theorem. The poles come from the denominator $\sinh (\pi \nu)$, and are at $i \nu+1=3+n$, with $n=0,1, \ldots$ This gives:

$$
g_{4}(z, \bar{z})=16 \pi^{3} D_{z, \bar{z}}\left[\sum_{n=0}^{\infty}(2 n+1)^{2} Q_{n}(\hat{z}) Q_{n}(\hat{\bar{z}})-\sum_{n=0}^{\infty}(2 n+2)^{2} Q_{n+\frac{1}{2}}(\hat{z}) Q_{n+\frac{1}{2}}(\hat{\bar{z}})\right]
$$

The square brackets above were computed in equation 4.18 of [1], in terms of the tree-level contact Witten diagram $g_{4}^{\text {contact }}(z, \bar{z})$ of scalars with scaling dimensions $\Delta_{i}=\left(1,1, \frac{3}{2}, \frac{3}{2}\right)$. Thus,

$$
g_{4}(z, \bar{z})=\frac{\pi}{8} D_{z, \bar{z}} g_{4}^{\text {contact }}(z, \bar{z})
$$

The finite coupling large- $N$ 4-point correlator of the conformal Gross-Neveu model on $A d S_{3}$, which is given by an infinite sum of fermionic bubble diagrams in figure 3 , was computed in terms of a scalar tree-level contact diagram $g_{4}^{\text {contact }}(z, \bar{z})$ ! In [1], the 4-point correlator in the conformal $O(N)$ model on $A d S_{3}$ was computed in terms of the same contact diagram $g_{4}^{\text {contact }}(z, \bar{z})$. It would be interesting to understand whether or not this is a coincidence.

\section{Ladder diagrams in AdS}

In this section we derive the spectral representation for AdS ladder diagrams, with an arbitrary number of rungs. We consider only scalars in this section. The ladder diagrams are written as a gluing of tree-level exchange 4-point diagrams. This is possible due to two properties: 1. The spectral/split representation of the bulk-to-bulk propagator. 2. The 6J-symbol expansion of a tree-level exchange diagram in cross channel conformal blocks. See $[66,76]$ 

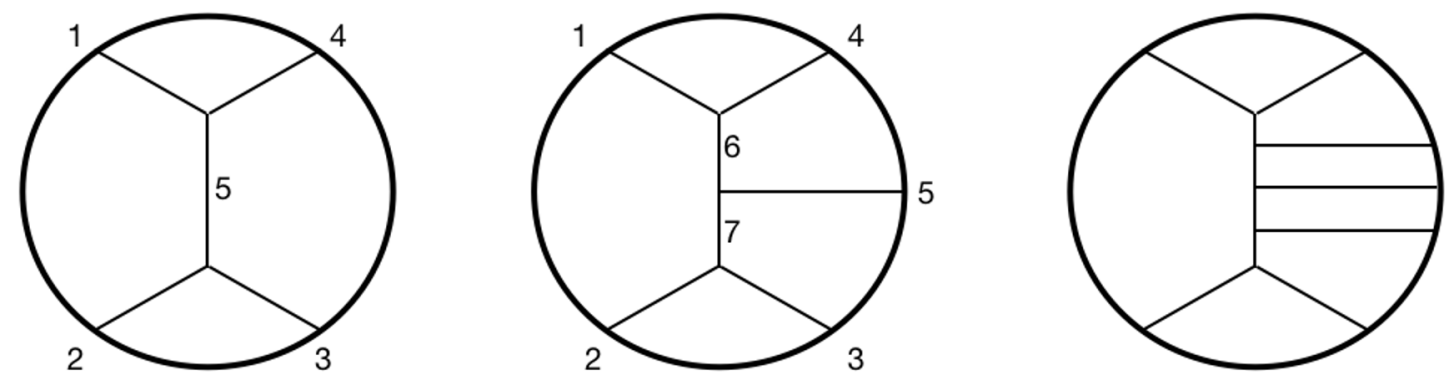

Figure 4. Left: 4-point tree-level exchange diagram. Middle: 5-point tree-level exchange diagram. Right: $N$-point tree-level exchange diagram in the comb channel.

\subsection{Warmup: tree-level comb Witten diagrams}

We consider here the comb channel tree-level diagrams in AdS, figure 4. The 4-point exchange Witten diagram, figure 4-Left, is given by:

$$
\begin{aligned}
\mathcal{A}_{5, \text { exch }}^{3214}= & \int d^{d+1} x_{1} d^{d+1} x_{2} G_{\Delta_{5}}\left(x_{1}, x_{2}\right) \\
& \times K_{\Delta_{1}}\left(P_{1}, x_{1}\right) K_{\Delta_{4}}\left(P_{4}, x_{1}\right) K_{\Delta_{3}}\left(P_{3}, x_{2}\right) K_{\Delta_{2}}\left(P_{2}, x_{2}\right)
\end{aligned}
$$

where $G_{\Delta_{5}}\left(x_{1}, x_{2}\right)$ is a bulk-to-bulk propagator. The spectral representation gives:

$$
\mathcal{A}_{5, \mathrm{exch}}^{3214}=\int_{-\infty}^{\infty} d \mu_{5} \frac{\pi \mu_{5}^{2}}{\mu_{5}^{2}+\left(\Delta_{5}-\frac{d}{2}\right)^{2}} b_{32 \mu_{5}} b_{-\mu_{5} 14} \Psi_{\mu_{5}}^{3214}
$$

where $b_{123}$ is defined in eq. (A.18), and the 4 -point conformal partial wave is:

$$
\Psi_{5}^{1234}=\int d^{d+1} x_{5}\left\langle\mathcal{O}_{1} \mathcal{O}_{2} \mathcal{O}_{5}\right\rangle\left\langle\tilde{\mathcal{O}}_{5} \mathcal{O}_{3} \mathcal{O}_{4}\right\rangle
$$

In a similar manner, the tree-level 5-point comb diagram, figure 4-Middle, gives:

$$
\mathcal{A}_{\text {tree }}^{(5)}=\int_{-\infty}^{\infty} \frac{d \mu_{6} d \mu_{7} \mu_{6}^{2} \mu_{7}^{2}}{\left[\mu_{6}^{2}+\left(\Delta_{6}-\frac{d}{2}\right)^{2}\right]\left[\mu_{7}^{2}+\left(\Delta_{7}-\frac{d}{2}\right)^{2}\right]} b_{12 \mu_{6}} b_{-\mu_{6}, 5,-\mu_{7}} b_{\mu_{7} 34} \Psi_{\mu_{6}, \mu_{7}}^{12345}
$$

where the 5-point conformal partial wave is:

$$
\Psi_{67}^{12345}=\int d^{d+1} x_{6} d^{d+1} x_{7}\left\langle\mathcal{O}_{1} \mathcal{O}_{2} \mathcal{O}_{6}\right\rangle\left\langle\tilde{\mathcal{O}}_{6} \mathcal{O}_{5} \tilde{\mathcal{O}}_{7}\right\rangle\left\langle\mathcal{O}_{7} \mathcal{O}_{3} \mathcal{O}_{4}\right\rangle
$$

One can extend these results to $N$-point tree-level comb diagrams, figure 4-Right. The result is:

$$
\mathcal{A}_{\text {tree }}^{(N)}=\int_{-\infty}^{\infty}\left(\prod_{\text {horiz. }} \frac{d \mu_{i} \mu_{i}^{2}}{\mu_{i}^{2}+\left(\Delta_{i}-\frac{d}{2}\right)^{2}}\right)\left(\prod_{\text {vertices }} b_{i j k}\right) \Psi^{(\mathrm{N}-\text { point })}
$$

where the $N$-point conformal partial wave is:

$$
\begin{aligned}
& \Psi_{N+1 \ldots 2 N-3}^{(\mathrm{N}-\text { point })} \\
& =\int d^{d+1} x_{N+1} \cdots d^{d+1} x_{2 N-3}\left\langle\mathcal{O}_{1} \mathcal{O}_{2} \mathcal{O}_{N+1}\right\rangle\left\langle\tilde{\mathcal{O}}_{N+1} \mathcal{O}_{3} \tilde{\mathcal{O}}_{N+2}\right\rangle \cdots\left\langle\mathcal{O}_{2 N-3} \mathcal{O}_{N-1} \mathcal{O}_{N}\right\rangle
\end{aligned}
$$



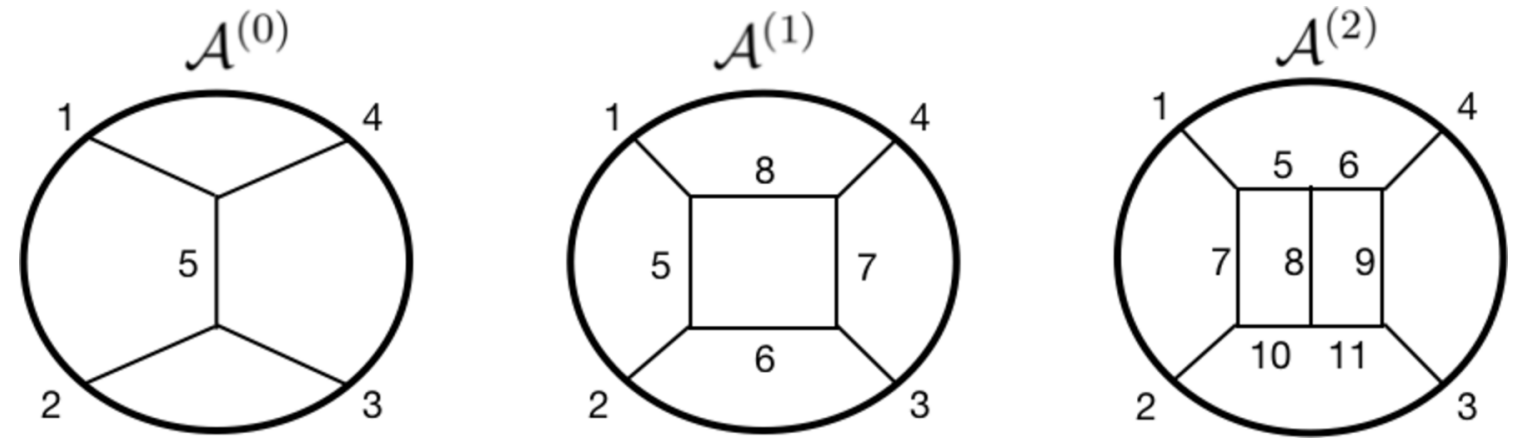

Figure 5. 4-point ladder diagrams. Left: the tree-level exchange diagram is the 0-loop ladder. Middle: the box diagram is the 1-loop ladder diagram. Right: the 2-loop ladder diagram.

\subsection{4-point ladder diagrams}

The exchange diagram (figure 5-Left) expanded in the direct channel is (eq. (4.2)):

$$
\mathcal{A}_{5, \mathrm{exch}}^{3214}=\int_{-\infty}^{\infty} d \mu_{5} \frac{\pi \mu_{5}^{2}}{\mu_{5}^{2}+\left(\Delta_{5}-\frac{d}{2}\right)^{2}} b_{32 \mu_{5}} b_{-\mu_{5} 14} \Psi_{\mu_{5}}^{3214}
$$

The same diagram can be expanded $[66,76]$ in the cross channel conformal partial waves $\Psi_{\mu, J}^{1234}$ :

$$
\mathcal{A}_{5, \text { exch }}^{3214}=b_{325} b_{\tilde{5} 14} \sum_{J=0}^{\infty} \int \frac{d \mu}{2 \pi i}\left(d-2 \Delta_{5}\right) K_{\tilde{5}}^{14}\left(\begin{array}{l}
\mathcal{O}_{1}, \mathcal{O}_{2}, \mathcal{O}_{5} \\
\mathcal{O}_{3}, \mathcal{O}_{4}, \mathcal{O}_{\mu, J}
\end{array}\right) \frac{1}{n_{\mathcal{O}_{\mu, J}}} \Psi_{\mu, J}^{1234}
$$

Let us define the factor appearing in the integrand above:

$$
\mathcal{J}_{\mathcal{O}_{5}, \mathcal{O}_{\mu, J}}^{1234} \equiv\left(d-2 \Delta_{5}\right) b_{325} b_{514} K_{\tilde{5}}^{14}\left(\begin{array}{l}
\mathcal{O}_{1}, \mathcal{O}_{2}, \mathcal{O}_{5} \\
\mathcal{O}_{3}, \mathcal{O}_{4}, \mathcal{O}_{\mu, J}
\end{array}\right) \frac{1}{n_{\mathcal{O}_{\mu, J}}}
$$

Therefore we can write:

$$
\mathcal{A}^{(0)}=\mathcal{A}_{5, \mathrm{exch}}^{3214}=\sum_{J=0}^{\infty} \int \frac{d \mu}{2 \pi i} \mathcal{J}_{\mathcal{O}_{5}, \mathcal{O}_{\mu, J}}^{1234} \Psi_{\mu, J}^{1234}
$$

This exchange diagram is the lowest order (zero loop) 4-point ladder diagram, figure 5-Left. In order to simplify the notation, we suppress writing the exchanged $\mathcal{O}_{5}$ operator, namely we write:

$$
\mathcal{J}_{\mu, J}^{1234} \equiv \mathcal{J}_{\mathcal{O}_{5}, \mathcal{O}_{\mu, J}}^{1234}
$$

The 1-loop ladder is the box diagram, figure 5-Middle. The box diagram can be computed as a gluing of two exchange diagrams:

$$
\mathcal{A}^{(1)}=\mathcal{A}_{\mathrm{Box}}=\mathcal{A}_{5, \mathrm{exch}}^{621 \tilde{\tilde{8}}} \otimes \mathcal{A}_{7, \text { exch }}^{3 \tilde{\tilde{6} 84}}
$$

Similarily, the 2-loop ladder (figure 5-Right) is a gluing of three exchange diagrams:

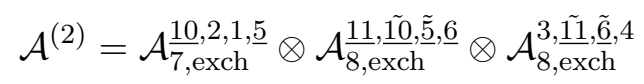


The $N$-loop ladder diagram is a gluing of $N+1$ exchange diagrams. Schematically:

$$
\mathcal{A}^{(N)}=\mathcal{A}_{1, \text { exch }} \otimes \mathcal{A}_{2, \text { exch }} \otimes \cdots \otimes \mathcal{A}_{N+1, \text { exch }}
$$

To derive an explicit expression for the $N$-loop ladder diagram, it is useful to write it as a conformal partial wave decomposition:

$$
\mathcal{A}^{(N)}=\sum_{J=0}^{\infty} \int_{-\infty}^{\infty} \frac{d \mu}{2 \pi i} C_{\mu, J}^{(N)} \Psi_{\mu, J}^{1234}
$$

where $C_{\mu, J}^{(N)}$ is the OPE function at $N$-loops, and $\Psi_{\mu, J}^{1234}$ is the 4-point conformal partial wave. For the tree-level exchange diagram we have from eq. (4.11):

$$
C_{\mu, J}^{(0)}=\mathcal{J}_{\mu, J}^{1,2,3,4}
$$

The 1-loop ladder OPE function is:

$$
C_{\mu, J}^{(1)}=B_{\mu, J} \int_{-\infty}^{\infty}\left(\prod_{i=6,8} \frac{d \nu_{i} \nu_{i}^{2}}{\nu_{i}^{2}+\left(\Delta_{i}-\frac{d}{2}\right)^{2}}\right) \mathcal{J}_{\mu, J}^{1,2,6,8} \mathcal{J}_{\mu, J}^{\tilde{8}, \tilde{6}, 3,4}
$$

We derive this in appendix A. For a somewhat different derivation, see [66]. $B_{\mu, J}$ is a conformal bubble factor. The external operators in $\mathcal{J}$ are highlighted in red. Notice that $i$ runs over the horizontal bulk-to-bulk propagators, see figure 5 .

In a similar fashion, one can derive the OPE function of the 2-loop ladder figure 5Right:

$$
C_{\mu, J}^{(2)}=\left(B_{\mu, J}\right)^{2} \int_{-\infty}^{\infty}\left(\prod_{i=5,6,10,11} \frac{d \nu_{i} \nu_{i}^{2}}{\nu_{i}^{2}+\left(\Delta_{i}-\frac{d}{2}\right)^{2}}\right) \mathcal{J}_{\mu, J}^{1,2,10,5} \mathcal{J}_{\mu, J}^{\tilde{5}, \tilde{1}, 11,6} \mathcal{J}_{\mu, J}^{\tilde{6}, \tilde{1}, 3,4}
$$

See also [66]. We see that there is a product of $\mathcal{J}$ 's, with their indices integrated over. Let us define the product:

$$
\prod_{\text {vertical }} \mathcal{J}_{\mu, J}=\mathcal{J}_{\mu, J}^{1,2, A_{1}, B_{1}} \mathcal{J}_{\mu, J}^{\tilde{B}_{1}, \tilde{A}_{1}, A_{2}, B_{2}} \mathcal{J}_{\mu, J}^{\tilde{B}_{2}, \tilde{A}_{2}, A_{3}, B_{3}} \cdots \mathcal{J}_{\mu, J}^{\tilde{B}_{\hat{N}-1}, \tilde{A}_{\hat{N}-1}, A_{\hat{N}}, B_{\hat{N}}} \mathcal{J}_{\mu, J}^{\tilde{B}_{\hat{N}}, \tilde{A}_{\hat{N}}, 3,4}
$$

The $N$-loop ladder diagram OPE function is given by:

$$
C_{\mu, J}^{(\mathrm{N}-\mathrm{loop})}=\left(B_{\mu, J}\right)^{N} \int_{-\infty}^{\infty}\left(\prod_{\text {horiz. }} \frac{d \nu_{i} \nu_{i}^{2}}{\nu_{i}^{2}+\left(\Delta_{i}-\frac{d}{2}\right)^{2}}\right) \prod_{\text {vertical }} \mathcal{J}_{\mu, J}
$$

Notice that all of the spectral integrals over the vertical propagators have been effectively performed, and we are left only with spectral integrals over the horizontal propagators. There is a $6 \mathrm{j}$-symbol factor $\mathcal{J}_{\mu, J}$ for each vertical bulk-to-bulk propagator. There is an integral $d \nu_{i}$ for each horizontal bulk-to-bulk propagators. 

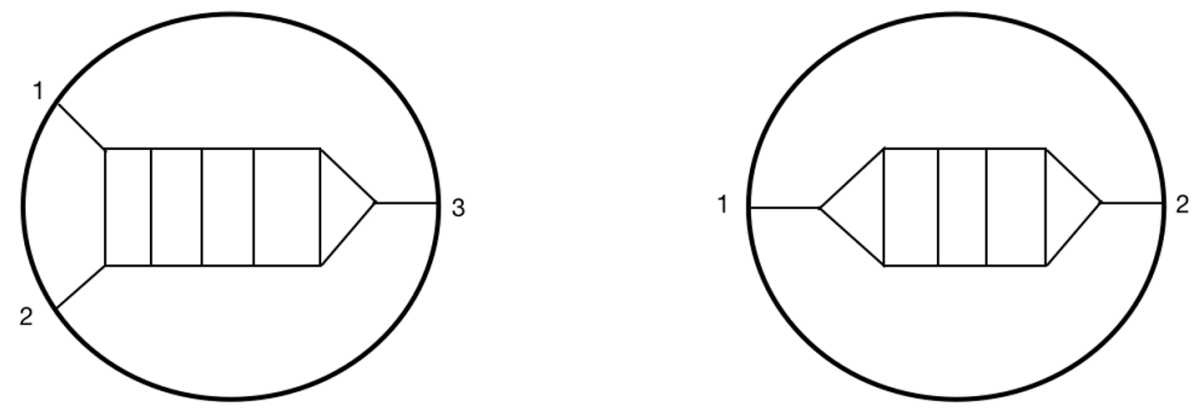

Figure 6. Left: an example of a 3-point ladder diagram. Right: an example of a 2-point ladder diagram.

\subsection{2 and 3-point ladder diagrams}

In the previous subsection we looked at 4-point ladder diagrams in AdS. In this subsection we consider ladder 3-point and 2-point functions, figure 6 . Thus the gluings for the 3-point ladder are:

$$
\mathcal{A}_{(3-\text { point })}^{(N)}=\mathcal{A}_{(3-\text { point })} \otimes \mathcal{A}_{1, \text { exch }} \otimes \cdots \otimes \mathcal{A}_{N, \text { exch }}
$$

and similarly for the 2-point functions:

$$
\mathcal{A}_{(2-\text { point })}^{(N)}=\mathcal{A}_{(3-\text { point })} \otimes \mathcal{A}_{1, \text { exch }} \otimes \cdots \otimes \mathcal{A}_{N-1, \text { exch }} \otimes \mathcal{A}_{(3-\text { point })}
$$

A computation similar to the 4-point case gives:

$$
\mathcal{A}_{(3-\text { point })}^{(N)}=C_{(3-\text { point })}^{(N)}\left\langle\mathcal{O}\left(P_{1}\right) \mathcal{O}\left(P_{2}\right) \mathcal{O}\left(P_{3}\right)\right\rangle
$$

and

$$
\mathcal{A}_{(2-\text { point })}^{(N)}=C_{(2-\text { point })}^{(N)}\left\langle\mathcal{O}\left(P_{1}\right) \mathcal{O}\left(P_{2}\right)\right\rangle
$$

Compared to the 4-point ladders of eq. (4.16), the 3-point ladders have fixed space-time dependence given by $\left\langle O\left(P_{1}\right) O\left(P_{2}\right) O\left(P_{3}\right)\right\rangle$. Similarly the 2-point ladders have 2-point structure $\left\langle\mathcal{O}\left(P_{1}\right) \mathcal{O}\left(P_{2}\right)\right\rangle$. The factors $C^{(N)}$ above depend on the scaling dimensions (and not on the space-time coordinates), and are given by:

$$
C_{(3-\text { point })}^{(\mathrm{N}-\text { loop })}=\left.\int_{-\infty}^{\infty}\left(\prod_{\text {horiz. }} \frac{d \nu_{i} \nu_{i}^{2}}{\nu_{i}^{2}+\left(\Delta_{i}-\frac{d}{2}\right)^{2}}\right)\left(B_{\mu, J}\right)^{N} \prod_{\text {vertical }} \mathcal{J}_{\mu, J}\right|_{J=0, \mu=\Delta_{3}-\frac{d}{2}}
$$

and

$$
C_{(2-\text { point })}^{(\mathrm{N}-\text { loop })}=\left.\int_{-\infty}^{\infty}\left(\prod_{\text {horiz. }} \frac{d \nu_{i} \nu_{i}^{2}}{\nu_{i}^{2}+\left(\Delta_{i}-\frac{d}{2}\right)^{2}}\right)\left(B_{\mu, J}\right)^{N} \prod_{\text {vertical }} \mathcal{J}_{\mu, J}\right|_{J=0, \mu=\Delta_{1}-\frac{d}{2}}
$$

\subsection{Mixed ladders/bubbles}

One can also compute diagrams that contain both ladders and bubbles. As an example, consider the diagram in figure 7-Left. It's OPE function is:

$$
\begin{aligned}
C_{\mu, J}= & \left(B_{\mu, J}\right)^{2} \frac{1}{\mu^{2}+\left(\Delta_{8}-\frac{d}{2}\right)^{2}} \frac{1}{\mu^{2}+\left(\Delta_{12}-\frac{d}{2}\right)^{2}} \widetilde{B}_{\mu}^{\left(\Delta_{13}, \Delta_{14}\right)} \\
& \times \int_{-\infty}^{\infty}\left(\prod_{i=5,6,10,11} \frac{d \nu_{i} \nu_{i}^{2}}{\nu_{i}^{2}+\left(\Delta_{i}-\frac{d}{2}\right)^{2}}\right) \mathcal{J}_{\mu, J}^{1,2,10,5} \mathcal{J}_{\mu, J}^{\tilde{5}, \tilde{10}, 6,11} b_{\tilde{6}, \tilde{1}, \mu} b_{\tilde{\mu}, 3,4}
\end{aligned}
$$



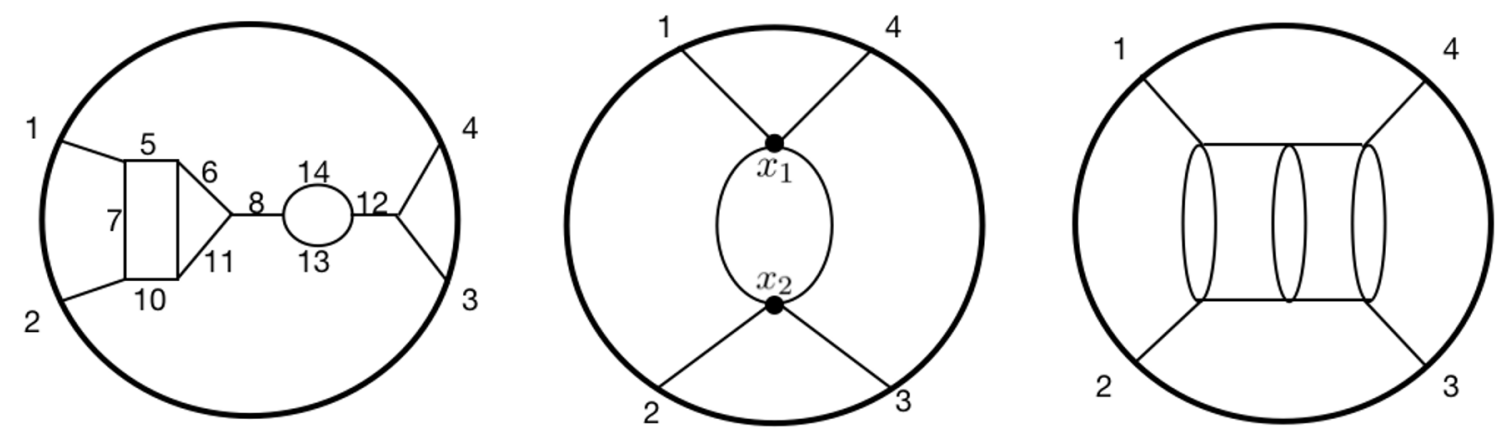

Figure 7. Left: an example of a mixed ladder-bubble diagram in $\phi^{3}$ theory on AdS. Middle: the 4-point 1-loop bubble diagram in $\phi^{4}$ theory. Right: an example of a $\phi^{4}$ ladder diagram.

where $\widetilde{B}_{\mu}^{\left(\Delta_{13}, \Delta_{14}\right)}$ is defined as the spectral representation of the 1-loop bubble:

$$
G_{\Delta_{13}}\left(x_{1}, x_{2}\right) G_{\Delta_{14}}\left(x_{1}, x_{2}\right)=\int_{-\infty}^{\infty} d \mu \widetilde{B}_{\mu}^{\left(\Delta_{13}, \Delta_{14}\right)} \Omega_{\mu}\left(x_{1}, x_{2}\right)
$$

where $\Omega_{\mu}\left(x_{1}, x_{2}\right)$ is the AdS harmonic function.

\section{5 $\phi^{4}$ ladders}

Let us consider ladder diagrams in $\phi^{4}$ theory on AdS. First consider the 4-point bubble diagram, figure 7-Middle:

$$
\mathcal{A}_{\text {bubble }}=\int d^{d+1} x_{1} d^{d+1} x_{2} K_{\Delta_{1}}\left(P_{1}, x_{1}\right) K_{\Delta_{2}}\left(P_{2}, x_{2}\right) K_{\Delta_{3}}\left(P_{3}, x_{2}\right) K_{\Delta_{4}}\left(P_{4}, x_{1}\right) G_{\Delta}^{2}\left(x_{1}, x_{2}\right)
$$

The bulk propagator squared $G_{\Delta}^{2}\left(x_{1}, x_{2}\right)$ can be expanded as a sum of bulk propagators [56, 57]:

$$
G_{\Delta}^{2}\left(x_{1}, x_{2}\right)=\sum_{n=0}^{\infty} a_{\Delta, \Delta}(n) G_{2 \Delta+2 n}\left(x_{1}, x_{2}\right)
$$

where the coefficients are:

$$
a_{\Delta_{1}, \Delta_{2}}(n)=\frac{\left(\frac{d}{2}\right)_{n}}{2 \pi^{\frac{d}{2}} \Gamma_{n+1}} \frac{\left(\Delta_{1}+\Delta_{2}+2 n\right)_{1-\frac{d}{2}}\left(\Delta_{1}+\Delta_{2}+n-d+1\right)_{n}}{\left(\Delta_{1}+n\right)_{1-\frac{d}{2}}\left(\Delta_{2}+n\right)_{1-\frac{d}{2}}\left(\Delta_{1}+\Delta_{2}+n-\frac{d}{2}\right)_{n}}
$$

Thus eq. (4.30) becomes:

$$
\begin{aligned}
& \mathcal{A}_{\text {bubble }}=\sum_{n=0}^{\infty} a_{\Delta, \Delta}(n) \\
& \times \int d^{d+1} x_{1} d^{d+1} x_{2} K_{\Delta_{1}}\left(P_{1}, x_{1}\right) K_{\Delta_{2}}\left(P_{2}, x_{2}\right) K_{\Delta_{3}}\left(P_{3}, x_{2}\right) K_{\Delta_{4}}\left(P_{4}, x_{1}\right) G_{2 \Delta+2 n}\left(x_{1}, x_{2}\right)
\end{aligned}
$$

The second line above is just a tree-level exchange diagram, see eq. (4.1).

$$
\mathcal{A}_{\text {bubble }}=\left.\sum_{n=0}^{\infty} a_{\Delta, \Delta}(n) \mathcal{A}_{\mathcal{O}^{\prime}, \operatorname{exch}}^{1234}\right|_{\Delta_{\mathcal{O}^{\prime}}=2 \Delta+2 n}
$$



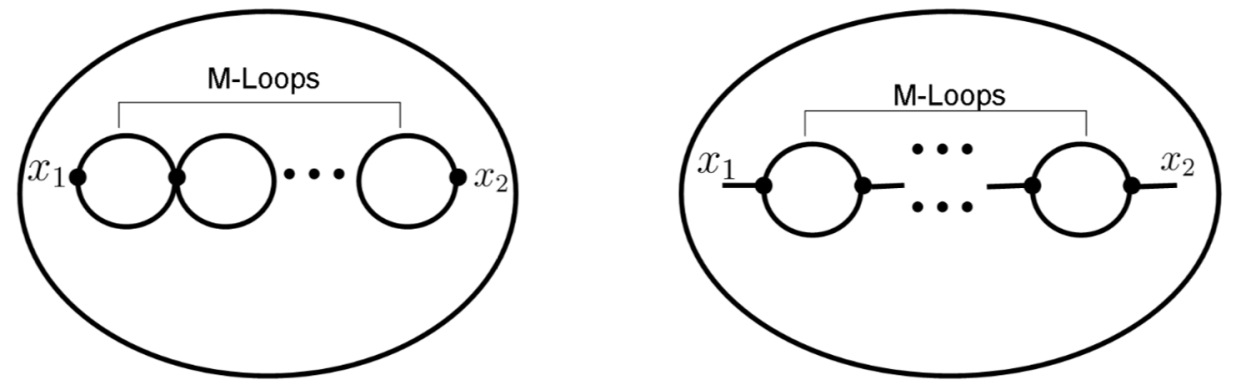

Figure 8. The type of bubble diagrams which we consider in section 5. Left: $\phi^{4}$ theory: the 2point bulk-to-bulk correlator consisting of a sequence of $M$-bubbles. Right: $\phi^{3}$ theory: the 2-point bulk-to-bulk correlator consisting of a sequence of $M$-bubbles.

Combining this with eqs. (4.16) and (4.17), gives the OPE function:

$$
\left(C_{\mu, J}^{(0)}\right)_{\text {bubble }}=\left.\sum_{n=0}^{\infty} a_{\Delta, \Delta}(n) C_{\mu, J}^{(0)}\right|_{\Delta_{\mathcal{O}^{\prime} \rightarrow 2 \Delta+2 n}}=\left.\sum_{n=0}^{\infty} a_{\Delta, \Delta}(n) \mathcal{J}_{\mathcal{O}^{\prime}, \mathcal{O}_{\mu, J}}^{1,2,4}\right|_{\Delta_{\mathcal{O}^{\prime} \rightarrow 2 \Delta+2 n}}
$$

Let us define the sum:

$$
\left.\mathcal{S}_{\mathcal{O}^{\prime}, \mathcal{O}_{\mu, J}}^{1,2,4} \equiv \sum_{n=0}^{\infty} a_{\Delta, \Delta}(n) \mathcal{J}_{\mathcal{O}^{\prime}, \mathcal{O}_{\mu, J}}^{1,2,3,4}\right|_{\Delta_{\mathcal{O}^{\prime}} \rightarrow 2 \Delta+2 n}
$$

Now can simply write eq. (4.35) as:

$$
\left(C_{\mu, J}^{(0)}\right)_{\text {bubble }}=\mathcal{S}_{\mathcal{O}^{\prime}, \mathcal{O}_{\mu, J}}^{1,2,4}
$$

One can easily extend this to higher loop $\phi^{4}$ ladders, as in figure 7-Right. All one has to do is make the replacement $\mathcal{J} \rightarrow \mathcal{S}$. Thus instead of eq. (4.21), we have the OPE function:

$$
\left(C_{\mu, J}^{(N)}\right)_{\text {bubble }}=\left(B_{\mu, J}\right)^{N} \int_{-\infty}^{\infty}\left(\prod_{\text {horiz. }} \frac{d \nu_{i} \nu_{i}^{2}}{\nu_{i}^{2}+\left(\Delta_{i}-\frac{d}{2}\right)^{2}}\right) \prod_{\text {vertical bubbles }} \mathcal{S}_{\mu, J}
$$

where the product is defined as:

$$
\prod_{\text {vertical bubbles }} \mathcal{S}_{\mu, J}=\mathcal{S}_{\mu, J}^{1,2, A_{1}, B_{1}} \mathcal{S}_{\mu, J}^{\tilde{B}_{1}, \tilde{A}_{1}, A_{2}, B_{2}} \mathcal{S}_{\mu, J}^{\tilde{B}_{2}, \tilde{A}_{2}, A_{3}, B_{3}} \ldots \mathcal{S}_{\mu, J}^{\tilde{B}_{\hat{N}-1}, \tilde{A}_{\hat{N}-1}, A_{\hat{N}}, B_{\hat{N}}} \mathcal{S}_{\mu, J}^{\tilde{B}_{\hat{N}}, \tilde{A}_{\hat{N}}, 3,4}
$$

\section{2-point bulk correlators in AdS}

Consider 2-point bulk bubble diagrams of scalars in $A d S_{3}$, with $x_{1}$ and $x_{2}$ being the external points in the bulk, such as those in figure 8. The $A d S_{d+1}$ bulk-to-bulk scalar propagator in position space is:

$$
G_{\Delta}\left(x_{1}, x_{2}\right)=\frac{\Gamma_{\Delta}}{2 \pi^{\frac{d}{2}} \Gamma_{\Delta-\frac{d}{2}+1}} \zeta^{-\Delta}{ }_{2} F_{1}\left(\Delta, \Delta-\frac{d-1}{2}, 2 \Delta-d+1,-4 \zeta^{-1}\right)
$$


where $\zeta=\frac{\left(z_{1}-z_{2}\right)^{2}+\left(\vec{x}_{1}-\vec{x}_{2}\right)^{2}}{z_{1} z_{2}}$ is the chordal distance squared between the points $x_{1}$ and $x_{2}$. When $d$ is even, the propagator above simplifies. In particular, for $d=2\left(A d S_{3}\right)$ :

$$
G_{\Delta}\left(x_{1}, x_{2}\right)=\frac{1}{2 \pi} \frac{1}{\sqrt{\zeta(\zeta+4)}}\left(\frac{2}{\sqrt{\zeta}+\sqrt{\zeta+4}}\right)^{2 \Delta-2}=\frac{1}{2 \pi} \frac{1}{\sqrt{\zeta(\zeta+4)}} \eta^{\Delta-1}
$$

i.e the bulk-to-bulk propagator is a power law in $\Delta$. We defined $\eta \equiv\left(\frac{2}{\sqrt{\zeta}+\sqrt{\zeta+4}}\right)^{2}$, which has the range $\eta$ is $0 \leq \eta \leq 1$. Likewise, in $A d S_{5}$ the bulk-to-bulk propagator becomes a power law in $\Delta$ :

$$
G_{\Delta}\left(x_{1}, x_{2}\right)=(A+B \Delta) \omega^{\Delta}
$$

where we defined:

$$
\begin{aligned}
& \omega \equiv 4 \zeta^{-1}\left(1+\sqrt{\frac{4+\zeta}{\zeta}}\right)^{-2} \\
& A \equiv \frac{\left(1+\sqrt{\frac{4+\zeta}{\zeta}}\right)^{3}}{64 \pi^{2}\left(\frac{4+\zeta}{\zeta}\right)^{\frac{3}{2}}}\left[-2\left(1+\sqrt{\frac{4+\zeta}{\zeta}}\right)+\frac{4}{\zeta}\left(-3+\sqrt{\frac{4+\zeta}{\zeta}}\right)\right] \\
& B \equiv \frac{\left(1+\sqrt{\frac{4+\zeta}{\zeta}}\right)^{3}}{64 \pi^{2}\left(\frac{4+\zeta}{\zeta}\right)^{\frac{3}{2}}}\left[2\left(1+\sqrt{\frac{4+\zeta}{\zeta}}\right)+\frac{8}{\zeta}\right]
\end{aligned}
$$

The spectral representation of the bulk-to-bulk propagator is:

$$
G_{\Delta}\left(x_{1}, x_{2}\right)=\int_{-\infty}^{\infty} \frac{d \nu}{\nu^{2}+\left(\Delta-\frac{d}{2}\right)^{2}} \Omega_{\nu}\left(x_{1}, x_{2}\right)
$$

where the AdS harmonic function is:

$$
\begin{aligned}
\Omega_{\nu}\left(x_{1}, x_{2}\right) & =\frac{i \nu}{2 \pi}\left(G_{\frac{d}{2}+i \nu}\left(x_{1}, x_{2}\right)-G_{\frac{d}{2}-i \nu}\left(x_{1}, x_{2}\right)\right) \\
& =\frac{h-\frac{d}{2}}{2 \pi}\left(G_{h}\left(x_{1}, x_{2}\right)-G_{d-h}\left(x_{1}, x_{2}\right)\right)
\end{aligned}
$$

where $h \equiv i \nu+\frac{d}{2}$.

$\boldsymbol{\phi}^{4}$ bulk bubble diagrams in $\boldsymbol{A d}_{\boldsymbol{5}}$. Consider a scalar field in AdS with $\phi^{4}$ interaction. The bubble diagrams figure 8-Left give a contribution to the 2-point function:

$$
\left\langle\phi^{2}\left(x_{1}\right) \phi^{2}\left(x_{2}\right)\right\rangle_{\text {bulk }}
$$

The spectral representation of a sequence of $M$ bubbles is just the $M$ th power of a single bubble.

$$
g_{2}^{(M)}\left(x_{1}, x_{2}\right)=\int_{-\infty}^{\infty} d \nu(\tilde{B}(\nu))^{M} \Omega_{\nu}\left(x_{1}, x_{2}\right)=\frac{1}{\pi} \int_{-\infty}^{\infty} d \nu(\tilde{B}(\nu))^{M} i \nu G_{\frac{d}{2}+i \nu}\left(x_{1}, x_{2}\right)
$$


where $G_{\frac{d}{2}+i \nu}\left(x_{1}, x_{2}\right)$ is the bulk-to-bulk propagator, and we used eq. (5.6). We go to position space by closing the $\nu$ contour and using the residue theorem. For a pole of order $M$ at $y=y_{0}$, the residue is:

$$
\left.\operatorname{Res}(F(y))\right|_{y=y_{0}}=\left.\frac{1}{\Gamma_{M}} \frac{d^{M-1}}{d y^{M-1}}\left[\left(y-y_{0}\right)^{M} F(y)\right]\right|_{y \rightarrow y_{0}}
$$

Thus eq. (5.8) gives:

$$
g_{2}^{(M)}\left(x_{1}, x_{2}\right)=\frac{2}{\Gamma_{M}} \sum_{n=0}^{\infty} \frac{d}{d^{M-1} h}\left[(h-(2 n+2 \Delta))^{M}(\tilde{B}(\nu))^{M}\left(h-\frac{d}{2}\right) G_{h}\left(x_{1}, x_{2}\right)\right]_{h \rightarrow(2 n+2 \Delta)}
$$

where $h \equiv \frac{d}{2}+i \nu$. As an example, consider the regularized bubble with $\Delta=2$ in $A d S_{5}$, eq. (C.8):

$$
\tilde{B}_{\text {reg. }}(\nu)=\frac{\nu^{3} \operatorname{coth}\left(\frac{\pi \nu}{2}\right)}{1+\nu^{2}}=\frac{(h-2)^{3} \cot \frac{\pi h}{2}}{(h-1)(h-3)}
$$

Plugging this in eq. (5.10) gives:

$$
g_{2}^{(M)}\left(x_{1}, x_{2}\right)=\frac{2}{\Gamma_{M}} \sum_{n=0}^{\infty} \frac{d}{d^{M-1} h}\left[\left((h-(2 n+4)) \frac{(h-2)^{3} \cot \frac{\pi h}{2}}{(h-1)(h-3)}\right)^{M}(h-2)(A+B h) \omega^{h}\right]_{h \rightarrow(2 n+4)}
$$

For a given value of $M$ (number of loops), one can compute the sum above in terms of hypergeometric functions. ${ }^{3}$

Fermionic bubble diagrams. Consider fermions in the bulk of $A d S_{3}(d=2)$. The poles of the 1-loop bubble are (see eq. 6.26 of [55]):

$$
\tilde{B}_{F}^{(d=2)}(\nu) \stackrel{1}{i \nu \sim 2 \Delta+2 n}-\frac{1}{i \nu-(2 \Delta+2 n)} \times \frac{(n+1)(2 \Delta+n-1)}{(2 \Delta+2 n)}
$$

Therefore for a chain of fermionic bubbles:

$$
\begin{aligned}
& g^{(M)}\left(x_{1}, x_{2}\right)=\int \frac{d \nu}{2 \pi}\left(\tilde{B}_{F}(\nu)\right)^{M} \Omega_{\nu}\left(x_{1}, x_{2}\right) \\
& =\sum_{n=0} \frac{d}{d^{M-1} h}\left[(h-(2 n+2 \Delta+1))^{M}\left(\tilde{B}_{F}(\nu)\right)^{M}(h-1) G_{h}\left(x_{1}, x_{2}\right)\right]_{h \rightarrow(2 n+2 \Delta)} \\
& =\frac{1}{2 \pi} \frac{1}{\sqrt{\zeta(\zeta+4)}} \sum_{n=0} \frac{d}{d^{M-1} h}\left[(h-(2 n+2 \Delta+1))^{M}\left(\tilde{B}_{F}(\nu)\right)^{M}(h-1) \eta^{h-1}\right]_{h \rightarrow(2 n+2 \Delta+1)}
\end{aligned}
$$

For $M=1$ computing the sum gives:

$$
g^{(M=1)}\left(x_{1}, x_{2}\right)=\frac{1}{2 \pi} \frac{1}{\sqrt{\zeta(\zeta+4)}} \frac{\eta^{2 \Delta}\left(1-2 \Delta+\eta^{2}(2 \Delta-3)\right)}{\left(\eta^{2}-1\right)^{3}}
$$

One can continue and compute higher loop bubble diagrams.

\footnotetext{
${ }^{3}$ We will leave it for the interested reader to obtain explicit expressions, which can easily be done with Mathematica. In a similar fashion, one can also compute bubble diagrams in $\phi^{3}$ theory, as in figure 8-Right.
} 

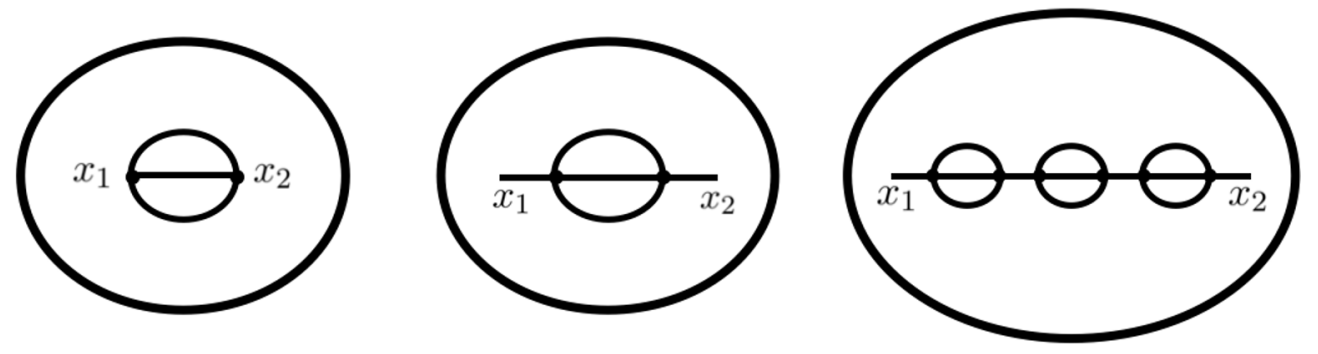

Figure 9. Sunset diagrams. Left: the sunset bubble. Middle: $\phi^{4}$ theory bulk 2-point function $\langle\phi \phi\rangle_{\text {bulk }}$ at 2-loops. Right: chain of sunset bubble diagrams in $\phi^{4}$.

Sunset bubbles. Consider the 2-loop bubble between 2-points in the bulk $x_{1}$ and $x_{2}$, otherwise known as the sunset diagram, see figure 9-Left. In position space this diagram is simply equal to the propagator cubed: $G_{\Delta}^{3}\left(x_{1}, x_{2}\right)$. In eq. 55 of [57], this was written in terms of an infinite sum over bulk propagators:

$$
G_{\Delta}^{3}\left(x_{1}, x_{2}\right)=\sum_{n=0}^{\infty} N_{3, \Delta}(n) G_{3 \Delta+2 n}\left(x_{1}, x_{2}\right)
$$

where

$$
N_{3, \Delta}(n)=\sum_{m=0}^{n} a_{\Delta, \Delta}(m) a_{\Delta, 2 \Delta+2 m}(n-m)
$$

and the coefficients $a_{\Delta_{1}, \Delta_{2}}$ are defined in eq. (4.32). Plugging eq. (5.5) in eq. (5.16) gives:

$$
\begin{aligned}
G_{\Delta}^{3}\left(x_{1}, x_{2}\right) & =\sum_{n=0}^{\infty} N_{3, \Delta}(n) G_{3 \Delta+2 n}\left(x_{1}, x_{2}\right) \\
& =\sum_{n=0}^{\infty} N_{3, \Delta}(n) \int_{-\infty}^{\infty} \frac{d \nu}{\nu^{2}+\left(3 \Delta+2 n-\frac{d}{2}\right)^{2}} \Omega_{\nu}\left(x_{1}, x_{2}\right) \\
& =\int_{-\infty}^{\infty} d \nu \Omega_{\nu}\left(x_{1}, x_{2}\right) \sum_{n=0}^{\infty} \frac{N_{3, \Delta}(n)}{\nu^{2}+\left(3 \Delta+2 n-\frac{d}{2}\right)^{2}} \\
& \equiv \int_{-\infty}^{\infty} d \nu \Omega_{\nu}\left(x_{1}, x_{2}\right) B_{\operatorname{sun}}(\nu)
\end{aligned}
$$

where $B_{\text {sun }}(\nu)$ is the spectral representation of $G_{\Delta}^{3}\left(x_{1}, x_{2}\right)$. Therefore the spectral representation of the sunset bubble is:

$$
B_{\text {sun }}(\nu)=\sum_{n=0}^{\infty} \frac{N_{3, \Delta}(n)}{\nu^{2}+\left(3 \Delta+2 n-\frac{d}{2}\right)^{2}}
$$

Now we focus on $A d S_{3}(d=2)$ where we have a simplification:

$$
a_{\Delta_{1}, \Delta_{2}}^{(d=2)}(n)=\frac{1}{2 \pi}
$$

thus

$$
N_{3, \Delta}^{(d=2)}(n)=\sum_{m=0}^{n} a_{\Delta, \Delta}(m) a_{\Delta, 2 \Delta+2 m}(n-m)=\frac{1}{4 \pi^{2}} \sum_{m=0}^{n} 1=\frac{n+1}{4 \pi^{2}}
$$


Therefore eq. (5.19) gives:

$$
B_{\text {sun }}^{(d=2)}(\nu)=\frac{1}{4 \pi^{2}} \sum_{n=0}^{\infty} \frac{n+1}{\nu^{2}+(3 \Delta+2 n-1)^{2}}
$$

This sum is logarithmically divergent and therefore should be regularized. Clearly the poles of the sunset bubble are:

$$
\tilde{B}_{\mathrm{sun}}^{(d=2)}(\nu) \stackrel{1+i \nu \sim 3 \Delta+2 n}{\sim}-\frac{1}{1+i \nu-(3 \Delta+2 n)} \times \frac{n+1}{(3 \Delta+2 n-1)}
$$

Consistency check. Let's compute the sum in eq. (5.16), and see that the result is consistent:

$$
\begin{aligned}
G_{\Delta}^{3}\left(x_{1}, x_{2}\right) & =\sum_{n=0}^{\infty} N_{3, \Delta}(n) G_{3 \Delta+2 n}\left(x_{1}, x_{2}\right) \\
& =\frac{1}{(2 \pi)^{3} \sqrt{\zeta(\zeta+4)}} \sum_{n=0}^{\infty}(n+1) \eta^{3 \Delta+2 n-1} \\
& =\left(\frac{1}{2 \pi} \frac{1}{\sqrt{\zeta(\zeta+4)}} \eta^{\Delta-1}\right)^{3}=G_{\Delta}^{3}\left(x_{1}, x_{2}\right)
\end{aligned}
$$

where in the first equality we used eqs. (5.2). The sum in the second line is just a geometric sum. We get the propagator cubed, and everything is consistent.

Chain of sunsets. Consider the 2-point bulk correlator composed of a chain of $M$ sunset bubbles such as that in figure 9-Right:

$$
g^{(M)}\left(x_{1}, x_{2}\right)=\int \frac{d \nu}{2 \pi} \frac{\left(\tilde{B}_{\mathrm{sun}}(\nu)\right)^{M}}{\left(\nu^{2}+\left(\delta-\frac{d}{2}\right)^{2}\right)^{M+1}} \Omega_{\nu}\left(x_{1}, x_{2}\right),
$$

For simplicity let's consider $A d S_{3}$ and $M=1$ (figure 9-Middle):

$$
\begin{aligned}
g^{(1)}\left(x_{1}, x_{2}\right)= & \int \frac{d \nu}{2 \pi} \frac{\tilde{B}_{\text {sun }}(\nu)}{((h+\delta-2)(h-\delta))^{2}} \Omega_{\nu}\left(x_{1}, x_{2}\right) \\
= & \frac{1}{2 \pi \sqrt{\zeta(\zeta+4)}} \sum_{n=0}^{\infty} \frac{(n+1) \eta^{3 \Delta+2 n-1}}{((2 n+3 \Delta+\delta-2)(2 n+3 \Delta-\delta))^{2}} \\
& +\left.\frac{1}{2 \pi} \frac{1}{\sqrt{\zeta(\zeta+4)}} \frac{d}{d h}\left[\frac{(h-1)}{(h+\delta-2)^{2}} \tilde{B}^{\text {sun }}(\nu) \eta^{h-1}\right]\right|_{h=\delta}
\end{aligned}
$$

where the last line above comes from the double pole $\sim \frac{1}{(h-\delta)^{2}}$. The last line can be computed by plugging $\tilde{B}^{\text {sun }}(\nu)$ from eq. (5.22). Note that one should first regularize the sum in eq. (5.22). 
The sum in the second line of eq. (5.26) can be computed as:

$$
\begin{aligned}
& \frac{1}{2 \pi \sqrt{\zeta(\zeta+4)}} \sum_{n=0}^{\infty} \frac{(n+1) \eta^{3 \Delta+2 n-1}}{((2 n+3 \Delta+\delta-2)(2 n+3 \Delta-\delta))^{2}} \\
& =\frac{1}{2 \pi \sqrt{\zeta(\zeta+4)}} \frac{\eta^{3 \Delta-1}}{32(\delta-1)^{3}}\left[6(\Delta-1) \Phi\left(\eta^{2}, 1, \frac{3 \Delta-\delta}{2}\right)-6(\Delta-1) \Phi\left(\eta^{2}, 1, \frac{3 \Delta+\delta-2}{2}\right)\right. \\
& \left.\quad+(\delta-1)\left((2+\delta-3 \Delta) \Phi\left(\eta^{2}, 2, \frac{3 \Delta-\delta}{2}\right)+(4-\delta-3 \Delta) \Phi\left(\eta^{2}, 2, \frac{3 \Delta+\delta-2}{2}\right)\right)\right]
\end{aligned}
$$

Where $\Phi$ is the Lerch transcendent function defined as:

$$
\Phi(y, s, \alpha) \equiv \sum_{n=0}^{\infty} \frac{y^{n}}{(n+\alpha)^{s}}
$$

\section{Acknowledgments}

I am grateful to Lorenzo Di Pietro, Shota Komatsu, Eric Perlmutter, David Meltzer, Alic Sivaramakrishnan for useful discussions.

\section{A Ladder diagram at 1-loop}

In this section we show the details of the calculation of the spectral representation of the 1-loop ladder diagram. The 4-point 1-loop ladder diagram is given by (see figure 5-Middle):

$$
\begin{aligned}
\mathcal{A}^{(1)}= & \int d^{d+1} x_{1} \cdots d^{d+1} x_{4} K_{\Delta_{1}}\left(P_{1}, x_{1}\right) K_{\Delta_{2}}\left(P_{2}, x_{2}\right) K_{\Delta_{3}}\left(P_{3}, x_{3}\right) K_{\Delta_{4}}\left(P_{4}, x_{4}\right) \\
& \times G_{\Delta_{5}}\left(x_{1}, x_{2}\right) G_{\Delta_{7}}\left(x_{3}, x_{4}\right) G_{\Delta_{8}}\left(x_{1}, x_{4}\right) G_{\Delta_{6}}\left(x_{2}, x_{3}\right) .
\end{aligned}
$$

We use the spectral representation of two of the bulk-to-bulk propagators

$$
\begin{aligned}
& G_{\Delta_{8}}\left(x_{1}, x_{4}\right)=\int_{-\infty}^{\infty} d \nu_{8} \frac{1}{\nu_{8}^{2}+\left(\Delta_{8}-\frac{d}{2}\right)^{2}} \Omega_{\nu_{8}}\left(x_{1}, x_{4}\right), \\
& G_{\Delta_{6}}\left(x_{2}, x_{3}\right)=\int_{-\infty}^{\infty} d \nu_{6} \frac{1}{\nu_{6}^{2}+\left(\Delta_{6}-\frac{d}{2}\right)^{2}} \Omega_{\nu_{6}}\left(x_{2}, x_{3}\right),
\end{aligned}
$$

and the split representation for AdS harmonic functions:

$$
\begin{aligned}
& \Omega_{\nu_{8}}\left(x_{1}, x_{4}\right)=\frac{\nu_{8}^{2}}{\pi} \int d^{d} Q_{8} K_{\frac{d}{2}-i \nu_{8}}\left(Q_{8}, x_{1}\right) K_{\frac{d}{2}+i \nu_{8}}\left(Q_{8}, x_{4}\right), \\
& \Omega_{\nu_{6}}\left(x_{2}, x_{3}\right)=\frac{\nu_{6}^{2}}{\pi} \int d^{d} Q_{6} K_{\frac{d}{2}+i \nu_{6}}\left(Q_{6}, x_{2}\right) K_{\frac{d}{2}-i \nu_{2}}\left(Q_{6}, x_{3}\right),
\end{aligned}
$$

where $Q_{i}$ are boundary points. This procedure is schematically illustrated in figure 10 . Therefore eq. (A.1) becomes:

$$
\begin{aligned}
& \mathcal{A}^{(1)}=\frac{1}{\pi^{2}} \prod_{i=6,8} \int_{-\infty}^{\infty} d \nu_{i} \int d^{d} Q_{i} \frac{\nu_{i}^{2}}{\nu_{i}^{2}+\left(\Delta_{i}-\frac{d}{2}\right)^{2}} \\
& \times \int d^{d+1} x_{1} d^{d+1} x_{2} K_{\Delta_{1}}\left(P_{1}, x_{1}\right) K_{\Delta_{2}}\left(P_{2}, x_{2}\right) K_{\frac{d}{2}-i \nu_{8}}\left(Q_{8}, x_{1}\right) K_{\frac{d}{2}+i \nu_{6}}\left(Q_{6}, x_{2}\right) G_{\Delta_{5}}\left(x_{1}, x_{2}\right) \\
& \times \int d^{d+1} x_{3} d^{d+1} x_{4} K_{\Delta_{3}}\left(P_{3}, x_{3}\right) K_{\Delta_{4}}\left(P_{4}, x_{4}\right) K_{\frac{d}{2}+i \nu_{8}}\left(Q_{8}, x_{4}\right) K_{\frac{d}{2}-i \nu_{6}}\left(Q_{6}, x_{3}\right) G_{\Delta_{7}}\left(x_{3}, x_{4}\right)
\end{aligned}
$$


The bottom 2 lines are tree-level exchange diagrams, thus:

$$
\mathcal{A}^{(1)}=\frac{1}{\pi^{2}} \prod_{i=6,8} \int_{-\infty}^{\infty} d \nu_{i} \int d^{d} Q_{i} \frac{\nu_{i}^{2}}{\nu_{i}^{2}+\left(\Delta_{i}-\frac{d}{2}\right)^{2}} \quad \mathcal{A}_{5, \mathrm{exch}}^{621 \tilde{8}} \otimes \mathcal{A}_{7, \mathrm{exch}}^{3 \tilde{6} 84}
$$

The exchange diagrams have a conformal partial wave expansion in the crossed channel (eq. (4.11)):

$$
\begin{aligned}
& \mathcal{A}_{\mathcal{O}_{5}, \text { exch }}^{621 \tilde{8}}=\sum_{J_{5}=0}^{\infty} \int \frac{d \mu_{5}}{2 \pi i} \mathcal{J}_{\mathcal{O}_{5}, O_{\mu_{5}, J_{5}}}^{126 \tilde{8}} \Psi_{\mu_{5}, J_{5}}^{126 \tilde{8}} \\
& \mathcal{A}_{\mathcal{O}_{7}, \text { exch }}^{3 \tilde{6} 84}=\sum_{J_{7}=0}^{\infty} \int \frac{d \mu_{7}}{2 \pi i} \mathcal{J}_{\mathcal{O}_{7}, O_{\mu_{7}, J_{7}}}^{8 \tilde{6} 34} \Psi_{\mu_{7}, J_{7}}^{8 \tilde{6} 34}
\end{aligned}
$$

Thus,

$$
\begin{aligned}
\mathcal{A}^{(1)}= & \frac{1}{\pi^{2}} \int_{-\infty}^{\infty}\left(\prod_{i=6,8} d \nu_{i} \frac{\nu_{i}^{2}}{\nu_{i}^{2}+\left(\Delta_{i}-\frac{d}{2}\right)^{2}}\right) \sum_{J_{5}=0}^{\infty} \sum_{J_{7}=0}^{\infty} \int \frac{d \mu_{5}}{2 \pi i} \int \frac{d \mu_{7}}{2 \pi i} \mathcal{J}_{\mathcal{O}_{5}, O_{\mu_{5}, J_{5}}}^{126 \tilde{8}} \mathcal{J}_{\mathcal{O}_{7}, O_{\mu_{7}, J_{7}}}^{8 \tilde{6} 34} \\
& \times \int d^{d} Q_{8} d^{d} Q_{6} \Psi_{\mu_{5}, J_{5}}^{126 \tilde{8}} \Psi_{\mu_{7}, J_{7}}^{8 \tilde{6} 34}
\end{aligned}
$$

The conformal partial waves have a shadow representation:

$$
\begin{aligned}
& \Psi_{\mu_{5}, J_{5}}^{126 \tilde{8}}=\int d^{d} P_{0}\left\langle O\left(P_{1}\right) O\left(P_{2}\right) O_{\mu_{5}, J_{5}}\left(P_{0}\right)\right\rangle\left\langle\tilde{O}_{\mu_{5}, J_{5}}\left(P_{0}\right) \tilde{O}\left(Q_{8}\right) O\left(Q_{6}\right)\right\rangle \\
& \Psi_{\mu_{7}, J_{7}}^{8 \tilde{6} 34}=\int d^{d} P_{0}^{\prime}\left\langle O\left(Q_{8}\right) \tilde{O}\left(Q_{6}\right) O_{\mu_{7}, J_{7}}\left(P_{0}^{\prime}\right)\right\rangle\left\langle\tilde{O}_{\mu_{7}, J_{7}}\left(P_{0}^{\prime}\right) O\left(P_{3}\right) O\left(P_{4}\right)\right\rangle
\end{aligned}
$$

Plugging this in eq. (A.9), we can perform the $Q_{8}, Q_{8}$ integrals as follows: ${ }^{4}$

$$
\begin{aligned}
& \int d^{d} Q_{8} d^{d} Q_{6}\left\langle\tilde{O}_{\mu_{5}, J_{5}}\left(P_{0}\right) \tilde{O}\left(Q_{8}\right) O\left(Q_{6}\right)\right\rangle\left\langle O\left(Q_{8}\right) \tilde{O}\left(Q_{6}\right) O_{\mu_{7}, J_{7}}\left(P_{0}^{\prime}\right)\right\rangle \\
& =B_{\mu_{5}, J_{5}} \delta_{P_{0}, P_{0}^{\prime}} \delta_{\mu_{5}, \mu_{7}} \delta_{J_{5}, J_{7}} .
\end{aligned}
$$

Thus:

$$
\int d^{d} Q_{8} d^{d} Q_{8} \Psi_{\mu_{5}, J_{5}}^{126 \tilde{8}} \Psi_{\mu_{7}, J_{7}}^{8 \tilde{6} 34}=B_{\mu_{5}, J_{5}} \Psi_{\mu_{5}, J_{5}}^{1234} \delta_{\mu_{5}, \mu_{7}} \delta_{J_{5}, J_{7}}
$$

and we get:

$$
\frac{1}{\pi^{2}} \sum_{J_{5}=0}^{\infty} \int \frac{d \mu_{5}}{2 \pi i}\left[B_{\mu_{5}, J_{5}} \int_{-\infty}^{\infty}\left(\prod_{i=6,8} d \nu_{i} \frac{\nu_{i}^{2}}{\nu_{i}^{2}+\left(\Delta_{i}-\frac{d}{2}\right)^{2}}\right) \mathcal{J}_{\mathcal{O}_{5}, O_{\mu_{5}, J_{5}}}^{126 \tilde{\mathcal{O}_{7}}} \mathcal{J}_{\mathcal{O}_{7}, O_{\mu_{5}, J_{5}}}^{8 \tilde{\sigma} 34}\right] \Psi_{\mu_{5}, J_{5}}^{1234}
$$

The external operators in $\mathcal{J}$ are highlighted in red. The factor in the square brackets above gives the OPE function:

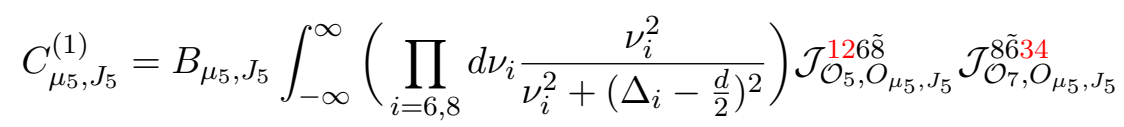

Thus we derived eq. (4.18).

\footnotetext{
${ }^{4}$ This is a CFT bubble integral. The bubble factor $B_{\mu_{5}, J_{5}}$ is a simple known function, defined e.g. in appendix A of [66].
} 


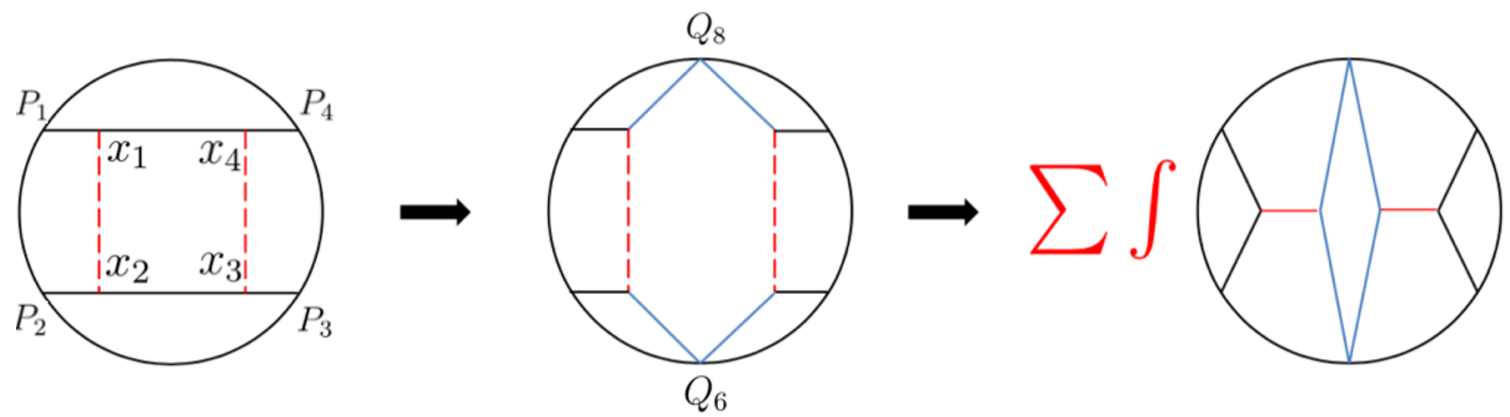

Figure 10. Schematic explanation of the computation of the one-loop box diagram. First, we rewrite the two horizontal bulk-to-bulk propagators using the split representation. The result is given by a convolution of two tree-level exchange diagrams. Second, we write exchange diagrams in the cross-channel conformal block expansion by using the $6 j$ symbol.

Conformal factors. Throughout this discussion of ladder diagrams we mainly use the notation of [66], see appendix A therein. The conformal 2-point function is:

$$
\left\langle\mathcal{O}_{\Delta, J}\left(P_{1}, z_{1}\right) \mathcal{O}_{\Delta, J}\left(P_{2}, z_{2}\right)\right\rangle=\mathcal{C}_{\Delta, J} \frac{\left(z_{1} \cdot I\left(P_{12}\right) \cdot z_{2}\right)^{J}}{P_{12}^{2 \Delta_{\mathcal{O}}}}
$$

with the definitions

$$
\begin{aligned}
\mathcal{C}_{\Delta} & \equiv \frac{\Gamma_{\Delta}}{2 \pi^{\frac{d}{2}} \Gamma_{\Delta+1-\frac{d}{2}}} \\
I_{\mu \nu}(x) & =\delta_{\mu \nu}-2 \frac{x_{\mu} x_{\nu}}{x^{2}}
\end{aligned}
$$

The AdS tree-level 3-point correlator:

$$
\int_{\mathrm{AdS}} d^{d+1} x K_{\Delta_{1}}\left(P_{1}, x\right) K_{\Delta_{2}}\left(P_{2}, x\right) K_{\Delta_{3}, J_{3}}\left(P_{3}, x\right)=b_{123}\left\langle\mathcal{O}_{1}\left(P_{1}\right) \mathcal{O}_{2}\left(P_{2}\right) \mathcal{O}_{3}\left(P_{3}\right)\right\rangle
$$

where we defined:

$$
b_{123} \equiv \mathcal{C}_{\Delta_{1}} \mathcal{C}_{\Delta_{2}} \mathcal{C}_{\Delta_{3}, J_{3}} \frac{\pi^{\frac{d}{2}} \Gamma_{\frac{\Delta_{1}+\Delta_{2}+\Delta_{3}+J_{3}-d}{2}} \Gamma_{\frac{-\Delta_{1}+\Delta_{2}+\Delta_{3}+J_{3}}{2}} \Gamma_{\frac{\Delta_{1}+\Delta_{2}-\Delta_{3}+J_{3}}{2}} \Gamma_{\frac{\Delta_{1}-\Delta_{2}+\Delta_{3}+J_{3}}{2}}}{2^{1-J_{3}} \Gamma_{\Delta_{1}} \Gamma_{\Delta_{2}} \Gamma_{\Delta_{3}+J_{3}}}
$$

\section{B An eigenvalue equation for ladder diagrams?}

Let us consider the 4-point ladder with all scaling dimensions equal to $\Delta$. Let's define $\nu_{0} \equiv \Delta-\frac{d}{2}$, and rewrite eq. (4.21):

$$
\frac{1}{\left(B_{\mu, J}\right)^{N}} C_{\mu, J}^{(\mathrm{N}-\text { loop })}=\left(B_{\mu, J}\right)^{N} \int_{-\infty}^{\infty}\left(\prod_{\text {horiz. }} \frac{d \nu_{i} \nu_{i}^{2}}{\nu_{i}^{2}+\left(\Delta-\frac{d}{2}\right)^{2}}\right) \prod_{\text {vertical }} \mathcal{J}_{\mu, J}
$$


Recalling eq. (4.20) we can view ${ }^{5}$ the equation above as a "matrix product" of $\mathcal{J}_{\mu, J}$ 's

$$
\frac{1}{\left(B_{\mu, J}\right)^{N}} C_{\mu, J}^{(\mathrm{N}-\text { loop })}=\Phi^{T} \cdot \widehat{\mathcal{J}}^{N-1} \cdot \Phi
$$

where

$$
\Phi_{\left(\nu_{1}, \nu_{2}\right)} \equiv \mathcal{J}_{\mu, J}^{-\nu_{2},-\nu_{1}, \nu_{0}, \nu_{0}}, \quad \Phi^{T\left(\nu_{1}, \nu_{2}\right)} \equiv \mathcal{J}_{\mu, J}^{\nu_{0}, \nu_{0}, \nu_{1}, \nu_{2}},
$$

is viewed as a vector and

$$
\widehat{\mathcal{J}}_{\left(\nu_{1}, \nu_{2}\right)}{ }^{\left(\nu_{3}, \nu_{4}\right)} \equiv \mathcal{J}_{\mu, J}^{-\nu_{2},-\nu_{1}, \nu_{3}, \nu_{4}},
$$

as a matrix, with indices given by couples of $\nu$ variables. The matrix multiplication is given by the integrals

$$
(\widehat{\mathcal{J}} \cdot \Phi)_{\left(\nu_{1}, \nu_{2}\right)} \equiv \int_{-\infty}^{\infty} \int_{-\infty}^{\infty} d \nu_{3} d \nu_{4} \frac{\nu_{3}^{2}}{\nu_{3}^{2}+\left(\Delta-\frac{d}{2}\right)^{2}} \frac{\nu_{4}^{2}}{\nu_{4}^{2}+\left(\Delta-\frac{d}{2}\right)^{2}} \widehat{\mathcal{J}}_{\left(\nu_{1}, \nu_{2}\right)}{ }^{\left(\nu_{3}, \nu_{4}\right)} \Phi_{\left(\nu_{3}, \nu_{4}\right)} .
$$

Suppose we can diagonalize the matrix $\widehat{\mathcal{J}}$, i.e. we find a "basis" (in some appropriate sense) of eigenvectors $\phi_{i}$ satisfying

$$
\left(\widehat{\mathcal{J}} \cdot \phi_{i}\right)_{\left(\nu_{1}, \nu_{2}\right)}=\lambda_{i} \phi_{i\left(\nu_{1}, \nu_{2}\right)},
$$

where $\lambda_{i}$ 's are the eigenvalues, and let us also assume that we can normalize the eigenvectors as

$$
\phi_{i}^{T} \cdot \phi_{j}=\delta_{i j} .
$$

We can then expand the vector $\Phi$ in this basis

$$
\Phi=\sum_{i} \alpha_{i} \phi_{i}
$$

In terms of these $\lambda_{i}$ 's and $\alpha_{i}{ }^{\prime}$ s, it is then immediate to write the OPE function ${ }^{6}$ of eq. (B.2):

$$
\frac{1}{\left(B_{\mu, J}\right)^{N}} C_{\mu, J}^{(\mathrm{N}-\text { loop })}=\Phi^{T} \cdot \widehat{\mathcal{J}}^{N-1} \cdot \Phi=\sum_{i}\left(\alpha_{i}\right)^{2} \lambda_{i}^{N-1}
$$

\section{The $O(N)$ model on $A d S_{5}$}

In this section we consider the large- $N O(N)$ model on $A d S_{5}$. The lagrangian of the $O(N)$ model is:

$$
\mathcal{L}=\frac{1}{2}\left(\partial \phi^{i}\right)^{2}+\frac{m^{2}}{2}\left(\phi^{i}\right)^{2}+\frac{\lambda}{2 N}\left(\phi^{i}\right)^{4}
$$

${ }^{5}$ I thank L. Di Pietro and S. Komatsu for initial collaboration on AdS ladder diagrams.

${ }^{6}$ The matrix product is:

$$
\begin{aligned}
\Phi^{T} \cdot \widehat{\mathcal{J}}^{N-1} \cdot \Phi & =\left(\sum_{i} \alpha_{i} \phi_{i}\right)^{T} \cdot \widehat{\mathcal{J}}^{N-1} \cdot\left(\sum_{j} \alpha_{j} \phi_{j}\right) \\
& =\sum_{i} \sum_{j} \alpha_{i} \alpha_{j} \lambda_{j}^{N-1} \phi_{i}^{T} \cdot \phi_{j}=\sum_{i} \sum_{j} \alpha_{i} \alpha_{j} \lambda_{j}^{N-1} \delta_{i j}=\sum_{i}\left(\alpha_{i}\right)^{2} \lambda_{i}^{N-1} .
\end{aligned}
$$


In contrast to the $A d S_{3}$ case $([1,55])$, in $A d S_{5}$ we will need to regularize the 1-loop bubble. The 4 -point correlator at large- $N$ is given by a resummation of bubble diagrams ${ }^{7}$ $([1,55])$ :

$$
g_{4}(z, \bar{z})=\int_{-\infty}^{\infty} d \nu \frac{1}{\lambda^{-1}+2 \tilde{B}(\nu)} \frac{\Gamma_{\Delta-\frac{d+2 i \nu}{4}}^{2} \Gamma_{\Delta-\frac{d-2 i \nu}{4}}^{2} \Gamma_{\frac{d+2 i \nu}{4}}^{4}}{\Gamma_{i \nu} \Gamma_{\frac{d}{2}+i \nu}} \mathcal{K}_{\frac{d}{2}+i \nu}(z, \bar{z})
$$

where $\tilde{B}(\nu)$ is the 1-loop bubble in the spectral representation. We will focus on the case of very strong coupling, in which $\lambda \rightarrow \infty$. $\tilde{B}(\nu)$ has single poles at (see e.g. eq. 4.24 of [55]):

$$
\tilde{B}(\nu) \stackrel{\frac{d}{2}+i \nu \sim 2 \Delta+2 n}{\sim}-\frac{1}{\frac{d}{2}+i \nu-(2 \Delta+2 n)} \frac{\left(\frac{d}{2}\right)_{n} \Gamma_{\Delta+n} \Gamma_{\Delta+n-\frac{d}{2}+\frac{1}{2}} \Gamma_{2 \Delta+n-\frac{d}{2}}}{2(4 \pi)^{\frac{d}{2}} \Gamma_{n+1} \Gamma_{\Delta+n+\frac{1}{2}} \Gamma_{\Delta+n-\frac{d}{2}+1} \Gamma_{2 \Delta-d+n+1}} .
$$

Focusing on $A d S_{5}$, we plug $d=4$ above and get:

$$
\tilde{B}(\nu) \stackrel{\frac{d}{2}+i \nu \sim 2 \Delta+2 n}{\sim}-\frac{1}{\frac{d}{2}+i \nu-(2 \Delta+2 n)} \times \frac{(n+1)(n+\Delta-1)(n+2 \Delta-3)}{2(2 \Delta+2 n-1)(2 \Delta+2 n-3)}
$$

To obtain $\tilde{B}(\nu)$, one should sum over all the poles in eq. (C.5). Since this sum is divergent, we regularize it by first subtracting the summand with $\nu=0$, and then sum over the poles:

$$
\tilde{B}_{\text {reg. }}(\nu) \equiv \sum_{n=0}^{\infty}\left[\text { eq. }(C .5)-\left(\text { eq. }\left.(C .5)\right|_{\nu=0}\right)\right]
$$

The regularized bubble $\tilde{B}_{\text {reg. }}(\nu)$ is finite. Performing the sum above gives:

$$
\tilde{B}_{\text {reg. }}(\nu)=\frac{(5-2 \Delta) \nu^{2}}{2\left(1+\nu^{2}\right)}+\frac{i \nu\left(\nu^{2}+4(\Delta-2)^{2}\right)}{4\left(1+\nu^{2}\right)}\left[\psi\left(-1+\Delta-\frac{i \nu}{2}\right)-\psi\left(-1+\Delta+\frac{i \nu}{2}\right)\right]
$$

Where $\psi(x)$ is the digamma function. For simplicity, let us focus on the case $\Delta=2$, in which the regularized bubble simplifies further:

$$
\tilde{B}_{\text {reg. }}(\nu)=\frac{\pi}{4} \frac{\nu^{3} \operatorname{coth}\left(\frac{\pi \nu}{2}\right)}{1+\nu^{2}}
$$

In $d=4$, the conformal block in eq. (C.3) can be written in terms of LegendreQ functions:

$$
\begin{aligned}
\mathcal{K}_{\beta}(z, \bar{z}) & =\frac{z \bar{z}}{\bar{z}-z}\left[k_{\beta-2}(z) k_{\beta}(\bar{z})-k_{\beta-2}(\bar{z}) k_{\beta}(z)\right] \\
& =\frac{z \bar{z}}{\bar{z}-z} 4 \frac{\Gamma(\beta) \Gamma(\beta-2)}{\Gamma^{2}\left(\frac{\beta-2}{2}\right) \Gamma^{2}\left(\frac{\beta}{2}\right)}\left[Q_{\frac{\beta}{2}-1}(\hat{z}) Q_{\frac{\beta}{2}-2}(\hat{\bar{z}})-Q_{\frac{\beta}{2}-2}(\hat{z}) Q_{\frac{\beta}{2}-1}(\hat{\bar{z}})\right]
\end{aligned}
$$

\footnotetext{
${ }^{7}$ The resummation of bubble diagrams is as in figure 3. In the spectral representation, the resummation gives a geometric sum:

$$
\lambda+\lambda^{2}(-2 \tilde{B}(\nu))+\lambda^{3}(-2 \tilde{B}(\nu))^{2}+\ldots=\frac{1}{\lambda^{-1}+2 \tilde{B}(\nu)}
$$

This explains the factor in eq. (C.3).
} 
Plugging this in eq. (C.3), one gets:

$$
g_{4}(z, \bar{z})=\frac{z \bar{z}}{\bar{z}-z} \int d \nu \frac{-\pi \nu\left(1+\nu^{2}\right)}{\sinh (\pi \nu)}\left[Q_{\frac{i \nu}{2}}(\hat{z}) Q_{\frac{i \nu}{2}-1}(\hat{\bar{z}})-Q_{\frac{i \nu}{2}}(\hat{\bar{z}}) Q_{\frac{i \nu}{2}-1}(\hat{z})\right]
$$

We rewrite the square brackets above, using the recurrence relation $Q_{\beta-1}(x)=(x+$ $\left.\frac{1-x^{2}}{\beta} \partial_{x}\right) Q_{\beta}(x)$ :

$$
Q_{\frac{i \nu}{2}}(\hat{z}) Q_{\frac{i \nu}{2}-1}(\hat{\bar{z}})-Q_{\frac{i \nu}{2}-1}(\hat{z}) Q_{\frac{i \nu}{2}}(\hat{\bar{z}})=\left[\frac{\hat{z}^{2}-1}{\frac{i \nu}{2}} \partial_{\hat{z}}-\frac{\hat{\bar{z}}^{2}-1}{\frac{i \nu}{2}} \partial_{\hat{\bar{z}}}+(\hat{\bar{z}}-\hat{z})\right] Q_{\frac{i \nu}{2}}(\hat{z}) Q_{\frac{i \nu}{2}}(\hat{\bar{z}})
$$

Thus

$$
g_{4}(z, \bar{z})=\frac{-\pi i z \bar{z}}{\bar{z}-z} \int d \nu \frac{i \nu(i \nu+1)(i \nu-1)}{\sinh (\pi \nu)}\left[\frac{\hat{z}^{2}-1}{\frac{i \nu}{2}} \partial_{\hat{z}}-\frac{\hat{\bar{z}}^{2}-1}{\frac{i \nu}{2}} \partial_{\hat{\bar{z}}}+(\hat{\bar{z}}-\hat{z})\right] Q_{\frac{i \nu}{2}}(\hat{z}) Q_{\frac{i \nu}{2}}(\hat{\bar{z}})
$$

Now we use the following identity for Legendre functions:

$$
\begin{aligned}
w_{z} Q_{\frac{\beta}{2}} & =\frac{\beta}{2}\left(\frac{\beta}{2}+1\right) Q_{\frac{\beta}{2}} \\
\text { where } \quad w_{z} & \equiv-\frac{d}{d \hat{z}}\left(1-\hat{z}^{2}\right) \frac{d}{d \hat{z}}
\end{aligned}
$$

Eq. (C.12) becomes:

$$
g_{4}(z, \bar{z})=\mathcal{P}_{1} \int d \nu \frac{(i \nu+1)}{\sinh (\pi \nu)} Q_{\frac{i \nu}{2}}(\hat{z}) Q_{\frac{i \nu}{2}}(\hat{\bar{z}})+\mathcal{P}_{2} \int d \nu \frac{(i \nu+1)^{2}}{\sinh (\pi \nu)} Q_{\frac{i \nu}{2}}(\hat{z}) Q_{\frac{i \nu}{2}}(\hat{\bar{z}})
$$

Where we have defined the differential operators:

$$
\begin{aligned}
& \mathcal{P}_{1}=-\pi i \frac{z \bar{z}}{\bar{z}-z}(\hat{\bar{z}}-\hat{z})\left(4 w_{z}+3\right)+4 \pi i \frac{z \bar{z}}{\bar{z}-z}\left[\left(\hat{z}^{2}-1\right) \partial_{\hat{z}}-\left(\hat{\bar{z}}^{2}-1\right) \partial_{\hat{\bar{z}}}\right] \\
& \mathcal{P}_{2} \equiv 3 \pi i \frac{z \bar{z}}{\bar{z}-z}(\hat{\bar{z}}-\hat{z})-2 \pi i \frac{z \bar{z}}{\bar{z}-z}\left[\left(\hat{z}^{2}-1\right) \partial_{\hat{z}}-\left(\hat{\bar{z}}^{2}-1\right) \partial_{\hat{\bar{z}}}\right]
\end{aligned}
$$

Using the residue theorem on eq. (C.14) gives:

$$
\begin{aligned}
g_{4}(z, \bar{z})= & \pi \mathcal{P}_{1}\left[\sum_{n=0}^{\infty}(2 n+2) Q_{n+\frac{1}{2}}(\hat{z}) Q_{n+\frac{1}{2}}(\hat{\bar{z}})-\sum_{n=0}^{\infty}(2 n+3) Q_{n+1}(\hat{z}) Q_{n+1}(\hat{\bar{z}})\right] \\
& +\pi \mathcal{P}_{2}\left[\sum_{n=0}^{\infty}(2 n+2)^{2} Q_{n+\frac{1}{2}}(\hat{z}) Q_{n+\frac{1}{2}}(\hat{\bar{z}})-\sum_{n=0}^{\infty}(2 n+3)^{2} Q_{n+1}(\hat{z}) Q_{n+1}(\hat{\bar{z}})\right] \\
= & \pi \mathcal{P}_{1} S_{6}+\pi \mathcal{P}_{2} S_{7}+\pi \mathcal{P}_{2} Q_{0}(\hat{z}) Q_{0}(\hat{\bar{z}})
\end{aligned}
$$

where we defined:

$$
\begin{aligned}
S_{6} & \equiv \sum_{n=0}^{\infty}(2 n+2) Q_{n+\frac{1}{2}}(\hat{z}) Q_{n+\frac{1}{2}}(\hat{\bar{z}})-\sum_{n=0}^{\infty}(2 n+3) Q_{n+1}(\hat{z}) Q_{n+1}(\hat{\bar{z}}) \\
S_{7} & \equiv \sum_{n=0}^{\infty}(2 n+2)^{2} Q_{n+\frac{1}{2}}(\hat{z}) Q_{n+\frac{1}{2}}(\hat{\bar{z}})-\sum_{n=0}^{\infty}(2 n+1)^{2} Q_{n}(\hat{z}) Q_{n}(\hat{\bar{z}})
\end{aligned}
$$



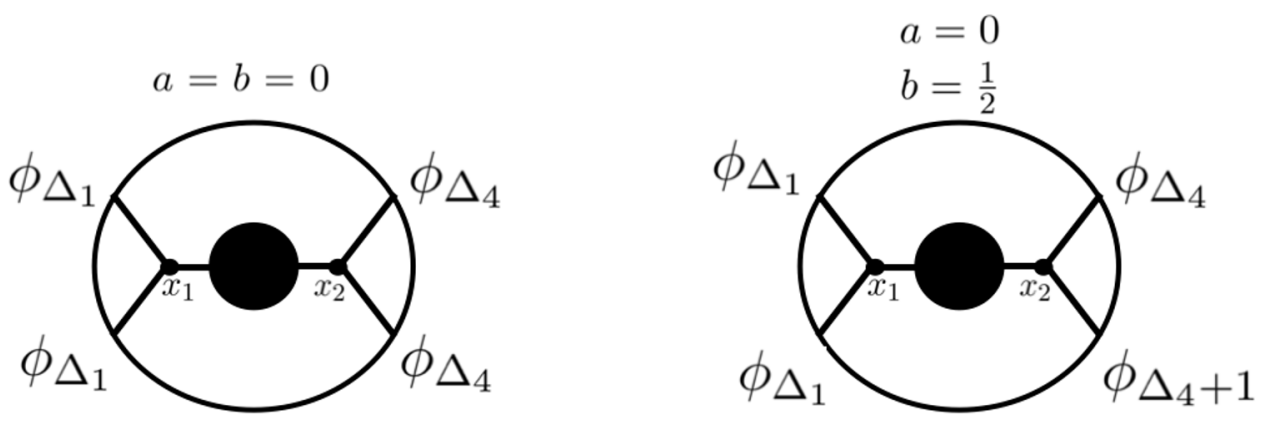

Figure 11. The black blob is a general bulk 2-point correlator. Left: the 4-point function with $a=b=0$. Right: the 4-point function with $a=0, b=\frac{1}{2}$, discussed in section D.

These sums can be computed analytically. The $S_{6}$ sum is equal to (see eq. 5.9 of [1]):

$$
\begin{aligned}
S_{6}= & {\left[2 Q_{\frac{1}{2}}(\hat{z}) Q_{\frac{1}{2}}(\hat{\bar{z}})+\frac{\frac{3}{2}}{(\hat{z}-\hat{\bar{z}})}\left(Q_{\frac{1}{2}}(\hat{z}) Q_{\frac{3}{2}}(\hat{\bar{z}})-Q_{\frac{3}{2}}(\hat{z}) Q_{\frac{1}{2}}(\hat{\bar{z}})\right)\right] } \\
& -\left[3 Q_{1}(\hat{z}) Q_{1}(\hat{\bar{z}})+\frac{2}{(\hat{z}-\hat{\bar{z}})}\left(Q_{1}(\hat{z}) Q_{2}(\hat{\bar{z}})-Q_{2}(\hat{z}) Q_{1}(\hat{\bar{z}})\right)\right]
\end{aligned}
$$

The sum $S_{7}$ is equal to (see eq. 4.18 of [1]):

$$
S_{7}=\frac{1}{128 \pi^{2}} g_{4}^{(\text {contact })}(\hat{z}, \hat{\bar{z}})
$$

where $g_{4}^{\text {(contact) }}(\hat{z}, \hat{\bar{z}})$ is defined as the scalar tree-level contact diagram in $A d S_{3}$ with external scaling dimensions $\Delta_{i}=\left(1,1, \frac{3}{2}, \frac{3}{2}\right)$.

To summarize, we have managed to compute analytically the (regularized) 4-point correlator of the $O(N)$ model on $A d S_{5}$, with scaling dimension $\Delta=2$ :

$$
g_{4}(z, \bar{z})=\pi \mathcal{P}_{1} S_{6}+\pi \mathcal{P}_{2} S_{7}+\pi \mathcal{P}_{2} Q_{0}(\hat{z}) Q_{0}(\hat{\bar{z}})
$$

where all of these functions were explicitly defined and computed above.

\section{Scalar 4-point bubble diagrams in $A d S_{5}$}

In this section we consider scalar 4-point bubble diagrams in $A d S_{5}$. We generalize the computations of [1] from $A d S_{3}$ to $A d S_{5}$. Consider the 4-point function in $d=4$ with external scaling dimensions $a \equiv \frac{\Delta_{2}-\Delta_{1}}{2}=0$ and $b \equiv \frac{\Delta_{3}-\Delta_{4}}{2}=\frac{1}{2}$, figure 11-Right. A simplification arises from the fact that the $d=4$ conformal block with $a=0$ and $b=\frac{1}{2}$ simplifies from a ${ }_{2} F_{1}$ to a power law:

$$
\begin{aligned}
\mathcal{K}_{\beta}^{\Delta_{i}}(z, \bar{z}) & =\frac{z \bar{z}}{z-\bar{z}}\left(z^{\frac{\beta}{2}}{ }_{2} F_{1}\left(\frac{\beta}{2}, \frac{\beta+1}{2}, \beta, z\right)\right) \times\left(\bar{z}^{\frac{\beta}{2}-1}{ }_{2} F_{1}\left(\frac{\beta-2}{2}, \frac{\beta-1}{2}, \beta-2, \bar{z}\right)\right)-(z \leftrightarrow \bar{z}) \\
& =\frac{z \bar{z}}{z-\bar{z}} \sqrt{\frac{u}{v}} \frac{2 \sqrt{z}}{1+\sqrt{1-z}}(4 Z)^{-2}(4 Z)^{\beta-1}-(z \leftrightarrow \bar{z}) \equiv \widetilde{Z} \sqrt{\frac{u}{v}}(4 Z)^{\beta-1}
\end{aligned}
$$


where the cross-ratios are $u=z \bar{z}$ and $v=(1-z)(1-\bar{z})$, and in the last line we defined:

$$
Z \equiv \frac{\sqrt{z \bar{z}}}{(1+\sqrt{1-z})(1+\sqrt{1-\bar{z}})}, \quad \widetilde{Z} \equiv \frac{2 \sqrt{z}}{1+\sqrt{1-z}}(4 Z)^{-2} \frac{z \bar{z}}{z-\bar{z}}-(z \leftrightarrow \bar{z})
$$

The spectral representation of a 4-point function (see e.g. eq. (2.18)) simplifies when plugging $\Delta_{2}=\Delta_{1}$ and $\Delta_{3}=\Delta_{4}+1$ :

$$
g_{4}(z, \bar{z})=\pi 2^{3-d} \int d \nu \tilde{F}_{\nu} \frac{\Gamma_{\frac{d}{2}+i \nu-1}}{4^{i \nu} \Gamma_{i \nu}}\left(\Gamma_{\Delta_{1}-\frac{d}{4}+\frac{i \nu}{2}} \Gamma_{\Delta_{1}-\frac{d}{4}-\frac{i \nu}{2}} \Gamma_{\Delta_{4}+\frac{1}{2}-\frac{d}{4}+\frac{i \nu}{2}} \Gamma_{\Delta_{4}+\frac{1}{2}-\frac{d}{4}-\frac{i \nu}{2}}\right) \mathcal{K}_{\frac{d}{2}+i \nu}^{\Delta_{i}}
$$

Putting $d=4$, and using eq. (D.1) gives:

$$
g_{4}(z, \bar{z})=2 \pi \sqrt{\frac{u}{v}} Z \widetilde{Z} \int_{-\infty}^{\infty} d \nu i \nu \tilde{F}_{\nu}\left(\Gamma_{\Delta_{1}-1+\frac{i \nu}{2}} \Gamma_{\Delta_{1}-1-\frac{i \nu}{2}} \Gamma_{\Delta_{4}-\frac{1}{2}+\frac{i \nu}{2}} \Gamma_{\Delta_{4}-\frac{1}{2}-\frac{i \nu}{2}}\right) Z^{i \nu}
$$

Rewriting this using a gamma function identity, $\Gamma_{x} \Gamma_{1-x}=\frac{\pi}{\sin (\pi x)}$, gives:

$$
g_{4}(z, \bar{z})=\sqrt{\frac{u}{v}} Z \widetilde{Z} \int d \nu \frac{2 \pi^{3} \tilde{F}_{\nu}}{\sin \pi\left(\Delta_{1}-1-\frac{i \nu}{2}\right) \sin \pi\left(\Delta_{4}-\frac{1}{2}-\frac{i \nu}{2}\right)} \frac{\Gamma_{\Delta_{4}-\frac{1}{2}+\frac{i \nu}{2}}}{\Gamma_{-\Delta_{4}+\frac{3}{2}+\frac{i \nu}{2}}} \frac{\Gamma_{\Delta_{1}-1+\frac{i \nu}{2}}}{\Gamma_{2-\Delta_{1}+\frac{i \nu}{2}}} Z^{i \nu}
$$

Now we close the contour and pick up poles from $\Gamma_{\Delta_{1}-1-\frac{i \nu}{2}}$ at $i \nu=2 \Delta_{1}-2+2 m$, and poles from $\Gamma_{\Delta_{4}-\frac{1}{2}-\frac{i \nu}{2}}$ at $i \nu=2 \Delta_{4}-1+2 m$ :

$$
\begin{aligned}
g_{4}(z, \bar{z}) \ni & \frac{8 \pi^{3} \sqrt{\frac{u}{v}} Z \widetilde{Z}}{\cos \pi\left(\Delta_{1}-\Delta_{4}\right)} \sum_{m=0}^{\infty} \frac{\Gamma_{\Delta_{1}+\Delta_{4}-\frac{3}{2}+m}}{\Gamma_{\Delta_{4}-\Delta_{1}+\frac{3}{2}+m}} \frac{\Gamma_{2 \Delta_{4}-1+m}}{\Gamma_{m+1}} \tilde{F}_{2 \Delta_{4}-1+2 m} Z^{2 \Delta_{4}-1+2 m} \\
& +\left(\Delta_{1} \leftrightarrow \Delta_{4}+\frac{1}{2}\right)
\end{aligned}
$$

These are contributions from the double-trace poles. As we will see, there can also be poles coming from $\tilde{F}_{\nu}$.

Double-discontinuity. We can also take the double-discontinuity of the 4-point correlator in eq. (D.5), which cancels the sine factors in the denominators: ${ }^{8}$

$$
d D i s c\left[g_{4}(z, \bar{z})\right]=2 \pi^{3} \sqrt{\frac{u}{v}} Z \widetilde{Z} \int d \nu \tilde{F}_{\nu} \frac{\Gamma_{\Delta_{4}-\frac{1}{2}+\frac{i \nu}{2}}}{\Gamma_{-\Delta_{4}+\frac{3}{2}+\frac{i \nu}{2}}} \frac{\Gamma_{\Delta_{1}-1+\frac{i \nu}{2}}}{\Gamma_{2-\Delta_{1}+\frac{i \nu}{2}}} Z^{i \nu}
$$

The double-discontinuity cancels the double-trace poles, and we are left just with the poles of the internal diagram (i.e poles of $\tilde{F}_{\nu}$ ). From the double-discontinuity, one can extract the full 4-point correlator by using the conformal dispersion relation [77]. Alternatively, one can extract the conformal data by plugging dDisc in the Lorenzian inversion formula [78].

$$
\begin{aligned}
& { }^{8} \text { Where we use } \\
& \qquad \operatorname{Disc}_{s}\left[\mathcal{K}_{\frac{d}{2}+i \nu}^{\Delta_{i}}(z, \bar{z})\right]=\sin \pi\left(\frac{\Delta_{1}+\Delta_{2}}{2}-\frac{i \nu+\frac{d}{2}}{2}\right) \sin \pi\left(\frac{\Delta_{3}+\Delta_{4}}{2}-\frac{i \nu+\frac{d}{2}}{2}\right) \mathcal{K}_{\frac{d}{2}+i \nu}^{\Delta_{i}}(z, \bar{z}) .
\end{aligned}
$$


Contact diagrams. The contact diagram has $\tilde{F}_{\nu}=1$, therefore eq. (D.6) gives:

$$
g_{4}(z, \bar{z})=\frac{8 \pi^{3} \sqrt{\frac{u}{v}} Z \widetilde{Z}}{\cos \pi\left(\Delta_{1}-\Delta_{4}\right)} \sum_{m=0}^{\infty} \frac{\Gamma_{\Delta_{1}+\Delta_{4}-\frac{3}{2}+m}}{\Gamma_{\Delta_{4}-\Delta_{1}+\frac{3}{2}+m}} \frac{\Gamma_{2 \Delta_{4}-1+m}}{\Gamma_{m+1}} Z^{2 \Delta_{4}-1+2 m}+\left(\Delta_{1} \leftrightarrow \Delta_{4}+\frac{1}{2}\right)
$$

This sum gives ${ }_{2} F_{1}$ 's:

$$
\begin{aligned}
g_{4}(z, \bar{z})= & \sqrt{\frac{u}{v}} \frac{\Gamma_{2 \Delta_{4}-1} \Gamma_{\Delta_{1}+\Delta_{4}-\frac{3}{2}} \widetilde{Z} Z^{2 \Delta_{4}}}{\left(8 \pi^{3}\right)^{-1} \cos \pi\left(\Delta_{1}-\Delta_{4}\right)}{ }_{2} F_{1}^{(\mathrm{reg})}\left(2 \Delta_{4}-1, \Delta_{1}+\Delta_{4}-\frac{3}{2}, \Delta_{4}-\Delta_{1}+\frac{3}{2}, Z^{2}\right) \\
& +\left(\Delta_{1} \leftrightarrow \Delta_{4}+\frac{1}{2}\right)
\end{aligned}
$$

where ${ }_{2} F_{1}^{(\mathrm{reg})}(a, b, c, x) \equiv \frac{1}{\Gamma_{c}} 2 F_{1}(a, b, c, x)$ is the regularized hypergeometric function.

Exchange diagrams. Consider the exchange diagram with internal scaling dimension $\Delta$, we plug $\tilde{F}_{\nu}=\frac{1}{\nu^{2}+(\Delta-2)^{2}}$ in eq. (D.4):

$$
g_{4}(z, \bar{z})=2 \pi \sqrt{\frac{u}{v}} Z \widetilde{Z} \int_{-\infty}^{\infty} d \nu \frac{\Gamma_{\Delta_{1}-1+\frac{i \nu}{2}} \Gamma_{\Delta_{1}-1-\frac{i \nu}{2}} \Gamma_{\Delta_{4}-\frac{1}{2}+\frac{i \nu}{2}} \Gamma_{\Delta_{4}-\frac{1}{2}-\frac{i \nu}{2}}}{\nu^{2}+(\Delta-2)^{2}} Z^{i \nu}
$$

One pole comes from the exchange operator at $i \nu=\Delta-2$, and there is also a tower of double-trace poles:

$$
g_{4}(z, \bar{z})=g_{\mathrm{exc}}(z, \bar{z})+g_{d . t}(z, \bar{z})
$$

where:

$$
g_{\mathrm{exc}}(z, \bar{z})=4 \pi^{2} \sqrt{\frac{u}{v}} \widetilde{Z} \frac{1}{2(\Delta-2)}\left(\Gamma_{\Delta_{1}-2+\frac{\Delta}{2}} \Gamma_{\Delta_{1}-\frac{\Delta}{2}} \Gamma_{\Delta_{4}-\frac{3}{2}+\frac{\Delta}{2}} \Gamma_{\Delta_{4}+\frac{1}{2}-\frac{\Delta}{2}}\right) Z^{\Delta-1}
$$

The contribution from the double-trace poles is:

$$
g_{d . t}(z, \bar{z})=\sqrt{\frac{u}{v}} \frac{8 \pi^{3} \widetilde{Z}}{\cos \pi\left(\Delta_{1}-\Delta_{4}\right)} \sum_{m=0}^{\infty} \frac{\frac{\Gamma_{\Delta_{1}+\Delta_{4}-\frac{3}{2}+m}}{\Gamma_{\Delta_{4}-\Delta_{1}+\frac{3}{2}+m}} \frac{\Gamma_{2 \Delta_{4}-1+m}}{\Gamma_{m+1}} Z^{2 \Delta_{4}+2 m}}{-\left(2 \Delta_{4}-1+2 m\right)^{2}+(\Delta-2)^{2}}+\left(\Delta_{1} \leftrightarrow \Delta_{4}+\frac{1}{2}\right)
$$

The result of the sum is:

$$
\begin{aligned}
& g_{d . t}(z, \bar{z})=8 \pi^{3} \sqrt{\frac{u}{v}} \widetilde{Z} \frac{\Gamma_{2 \Delta_{4}-1} \Gamma_{\Delta_{1}+\Delta_{4}-\frac{3}{2}} Z^{2 \Delta_{4}}}{4(\Delta-2) \cos \pi\left(\Delta_{1}-\Delta_{4}\right)} \\
& \times\left[\Gamma_{\Delta_{4}+\frac{\Delta}{2}-\frac{3}{2} 3} F_{2}^{(\mathrm{reg})}\left(2 \Delta_{4}-1, \Delta_{4}+\frac{\Delta}{2}-\frac{3}{2}, \Delta_{1}+\Delta_{4}-\frac{3}{2}: \Delta_{4}+\frac{\Delta}{2}-\frac{1}{2}, \frac{3}{2}+\Delta_{4}-\Delta_{1}, Z^{2}\right)\right. \\
& \left.-\Gamma_{\Delta_{4}-\frac{\Delta}{2}+\frac{1}{2} 3} F_{2}^{(\mathrm{reg})}\left(2 \Delta_{4}-1, \Delta_{4}-\frac{\Delta}{2}+\frac{1}{2}, \Delta_{1}+\Delta_{4}-\frac{3}{2}: \frac{3}{2}-\frac{\Delta}{2}+\Delta_{4}, \frac{3}{2}+\Delta_{4}-\Delta_{1}, Z^{2}\right)\right] \\
& +\left(\Delta_{1} \leftrightarrow \Delta_{4}+\frac{1}{2}\right)
\end{aligned}
$$


Loop diagrams. One can continue and compute loop bubble diagrams in $d=4$, just like we did in the case of $d=2$, [1]. For example, one can use the regularized bubble with $\Delta=2$ :

$$
\tilde{B}_{\text {reg. }}(\nu)=\frac{\pi}{4} \frac{\nu^{3} \operatorname{coth}\left(\frac{\pi \nu}{2}\right)}{1+\nu^{2}}=\frac{\pi}{4} \frac{(h-2)^{3} \cot \frac{\pi h}{2}}{(h-1)(h-3)}
$$

See eq. (C.8). For a diagram composed of a sequence of $M$ bubbles, we can plug $\tilde{F}_{\nu}=$ $(\tilde{B}(\nu))^{M}$ in eq. (D.4):

$$
g_{4}(z, \bar{z})=2 \pi \sqrt{\frac{u}{v}} Z \widetilde{Z} \int d \nu i \nu\left(\tilde{B}_{\mathrm{reg} .}(\nu)\right)^{M}\left(\Gamma_{\Delta_{1}-1+\frac{i \nu}{2}} \Gamma_{\Delta_{1}-1-\frac{i \nu}{2}} \Gamma_{\Delta_{4}-\frac{1}{2}+\frac{i \nu}{2}} \Gamma_{\Delta_{4}-\frac{1}{2}-\frac{i \nu}{2}}\right) Z^{i \nu}
$$

Closing the contour, the sums can be computed in terms of hypergeometric functions.

\section{E Scalar 4-point bubble diagrams in $A d S_{2}$}

Consider the 4-point function of scalar bubble diagrams in $A d S_{2}$ with equal external and internal scaling dimensions $\Delta$. We start by writing the spectral representation of the diagram in figure 11-Left:

$$
g_{4}(z)=\int d \nu \tilde{F}(\nu) \frac{\Gamma_{\Delta-\frac{d+2 i \nu}{4}}^{2} \Gamma_{\Delta-\frac{d-2 i \nu}{4}}^{2} \Gamma_{\frac{d+2 i \nu}{4}}^{4}}{\Gamma_{i \nu} \Gamma_{\frac{d}{2}+i \nu}} \mathcal{K}_{\frac{d}{2}+i \nu}(z),
$$

The conformal block in $d=1$ is:

$$
\mathcal{K}_{\beta}^{(d=1)}(z)=z^{\beta}{ }_{2} F_{1}(\beta, \beta, 2 \beta, z)=\frac{2 \Gamma_{2 \beta}}{\Gamma_{\beta}^{2}} Q_{\beta-1}(\hat{z})
$$

The poles of the 1-loop bubble in $d=1$ are:

$$
2 \tilde{B}(\nu) \stackrel{\frac{1}{2}+i \nu \sim 2 \Delta+2 n}{\sim}-\frac{1}{\frac{1}{2}+i \nu-(2 \Delta+2 n)} \frac{\Gamma_{n+\frac{1}{2}} \Gamma_{\Delta+n}^{2} \Gamma_{2 \Delta+n-\frac{1}{2}}}{\sqrt{\pi} \Gamma_{n+1} \Gamma_{\Delta+n+\frac{1}{2}}^{2} \Gamma_{2 \Delta+n}} .
$$

The spectral representation for the $M$ bubble diagram is $\tilde{F}(\nu)=(\tilde{B}(\nu))^{M}$, so we have in $d=1$ :

$$
g_{4}(z)=\sum_{n=0}^{\infty} \frac{d^{M+1}}{d n^{M+1}}\left(\left(\frac{\Gamma_{n+\frac{1}{2}} \Gamma_{\Delta+n}^{2} \Gamma_{2 \Delta+n-\frac{1}{2}}}{\sqrt{\pi} \Gamma_{n+1} \Gamma_{\Delta+n+\frac{1}{2}}^{2} \Gamma_{2 \Delta+n}}\right)^{M} \frac{\Gamma_{\Delta+n}^{2} \Gamma_{2 \Delta+n-\frac{1}{2}}^{2}}{\Gamma_{n+1}^{2} \Gamma_{\Delta+n+\frac{1}{2}}^{2}}(4 \Delta+4 n-1) Q_{2 \Delta+2 n-1}(\hat{z})\right)
$$

For simplicity let's consider $\Delta=1$. The contact diagram $M=0$ gives:

$$
g_{4}(z)=4 \sum_{n=0}^{\infty} \frac{d}{d n}\left((4 n+3) Q_{2 n+1}(\hat{z})\right)
$$

The result of this sum gives:

$$
g_{4}(z)=2 z^{2}\left(\frac{\log (1-z)}{z}+\frac{\log (z)}{1-z}\right)
$$

which precisely matches eq. 7.29 of [79]. 
One can also compute the 1-loop bubble, i.e $M=1$ and $\Delta=1$. From eq. (E.4) we have:

$$
g_{4}(z)=\sum_{n=0}^{\infty} \frac{d^{2}}{d n^{2}}\left(\frac{4 n+3}{(2 n+2)(2 n+1)} Q_{2 n+1}(\hat{z})\right)
$$

Using eq. (C.13), we can also write this as follows:

$$
w_{z} g_{4}(z)=\sum_{n=0}^{\infty} \frac{d^{2}}{d n^{2}}\left((4 n+3) Q_{2 n+1}(\hat{z})\right)
$$

where the differential operator is defined as $w_{z} \equiv-\frac{d}{d \hat{z}}\left(1-\hat{z}^{2}\right) \frac{d}{d \hat{z}}$. The r.h.s. of eq. (E.8) has a canonical form, and it would be interesting to see if it can be computed e.g. from eq. 5.9 of [1]. It would be interesting to compare the result with that of eq. 7.34 of [79].

Open Access. This article is distributed under the terms of the Creative Commons Attribution License (CC-BY 4.0), which permits any use, distribution and reproduction in any medium, provided the original author(s) and source are credited.

\section{References}

[1] D. Carmi, Loops in AdS: from the spectral representation to position space, JHEP 06 (2020) 049 [arXiv: 1910 . 14340] [INSPIRE].

[2] J.M. Maldacena, The large $N$ limit of superconformal field theories and supergravity, Int. J. Theor. Phys. 38 (1999) 1113 [hep-th/9711200] [INSPIRE].

[3] S.S. Gubser, I.R. Klebanov and A.M. Polyakov, Gauge theory correlators from noncritical string theory, Phys. Lett. B 428 (1998) 105 [hep-th/9802109] [InSPIRE].

[4] E. Witten, Anti-de Sitter space and holography, Adv. Theor. Math. Phys. 2 (1998) 253 [hep-th/9802150] [INSPIRE].

[5] C.G. Callan, Jr. and F. Wilczek, Infrared behavior at negative curvature, Nucl. Phys. B $\mathbf{3 4 0}$ (1990) 366 [INSPIRE].

[6] P. Breitenlohner and D.Z. Freedman, Stability in gauged extended supergravity, Annals Phys. 144 (1982) 249 [INSPIRE].

[7] O. Aharony, S.S. Gubser, J.M. Maldacena, H. Ooguri and Y. Oz, Large N field theories, string theory and gravity, Phys. Rept. 323 (2000) 183 [hep-th/9905111] [INSPIRE].

[8] O. Aharony, D. Marolf and M. Rangamani, Conformal field theories in anti-de Sitter space, JHEP 02 (2011) 041 [arXiv: 1011.6144] [INSPIRE].

[9] O. Aharony, M. Berkooz, D. Tong and S. Yankielowicz, Confinement in anti-de Sitter space, JHEP 02 (2013) 076 [arXiv: 1210.5195] [INSPIRE].

[10] C.J.C. Burges, D.Z. Freedman, S. Davis and G.W. Gibbons, Supersymmetry in anti-de Sitter space, Annals Phys. 167 (1986) 285 [INSPIRE].

[11] E. Witten, Anti-de Sitter space, thermal phase transition, and confinement in gauge theories, Adv. Theor. Math. Phys. 2 (1998) 505 [hep-th/9803131] [InSPIRE].

[12] C.P. Burgess and C.A. Lütken, Propagators and effective potentials in anti-de Sitter space, Phys. Lett. B 153 (1985) 137 [INSPIRE]. 
[13] T. Inami and H. Ooguri, Nambu-Goldstone bosons in curved space-time, Phys. Lett. B 163 (1985) 101 [INSPIRE].

[14] H. Liu, Scattering in anti-de Sitter space and operator product expansion, Phys. Rev. D 60 (1999) 106005 [hep-th/9811152] [INSPIRE].

[15] H. Liu and A.A. Tseytlin, On four point functions in the CFT/AdS correspondence, Phys. Rev. D 59 (1999) 086002 [hep-th/9807097] [INSPIRE].

[16] F.A. Dolan and H. Osborn, Conformal four point functions and the operator product expansion, Nucl. Phys. B 599 (2001) 459 [hep-th/0011040] [INSPIRE].

[17] D.Z. Freedman, S.D. Mathur, A. Matusis and L. Rastelli, Correlation functions in the $C F T_{d} / A d S_{d+1}$ correspondence, Nucl. Phys. B 546 (1999) 96 [hep-th/9804058] [InSPIRE].

[18] E. D'Hoker and D.Z. Freedman, General scalar exchange in AdS $S_{d+1}$, Nucl. Phys. B 550 (1999) 261 [hep-th/9811257] [INSPIRE].

[19] D.Z. Freedman, S.D. Mathur, A. Matusis and L. Rastelli, Comments on 4 point functions in the CFT/AdS correspondence, Phys. Lett. B 452 (1999) 61 [hep-th/9808006] [INSPIRE].

[20] E. D'Hoker and D.Z. Freedman, Gauge boson exchange in AdS $S_{d+1}$, Nucl. Phys. B 544 (1999) 612 [hep-th/9809179] [INSPIRE].

[21] E. D'Hoker, D.Z. Freedman and L. Rastelli, AdS/CFT four point functions: how to succeed at z-integrals without really trying, Nucl. Phys. B 562 (1999) 395 [hep-th/9905049] [INSPIRE].

[22] X. Zhou, Recursion relations in Witten diagrams and conformal partial waves, JHEP 05 (2019) 006 [arXiv:1812.01006] [INSPIRE].

[23] E. Hijano, P. Kraus, E. Perlmutter and R. Snively, Witten diagrams revisited: the AdS geometry of conformal blocks, JHEP 01 (2016) 146 [arXiv:1508.00501] [INSPIRE].

[24] J. Penedones, Writing CFT correlation functions as AdS scattering amplitudes, JHEP 03 (2011) 025 [arXiv: 1011.1485] [INSPIRE].

[25] A.L. Fitzpatrick, J. Kaplan, J. Penedones, S. Raju and B.C. van Rees, A natural language for AdS/CFT correlators, JHEP 11 (2011) 095 [arXiv: 1107.1499] [INSPIRE].

[26] M.F. Paulos, Towards Feynman rules for Mellin amplitudes, JHEP 10 (2011) 074 [arXiv:1107.1504] [INSPIRE].

[27] L. Rastelli and X. Zhou, How to succeed at holographic correlators without really trying, JHEP 04 (2018) 014 [arXiv:1710.05923] [INSPIRE].

[28] L. Rastelli and X. Zhou, Mellin amplitudes for $A d S_{5} \times S^{5}$, Phys. Rev. Lett. 118 (2017) 091602 [arXiv: 1608.06624] [INSPIRE].

[29] C. Cardona, Mellin-(Schwinger) representation of one-loop Witten diagrams in AdS, arXiv: 1708.06339 [INSPIRE].

[30] E.Y. Yuan, Loops in the bulk, arXiv:1710.01361 [INSPIRE].

[31] E.Y. Yuan, Simplicity in AdS perturbative dynamics, arXiv:1801.07283 [INSPIRE].

[32] S. Raju, BCFW for Witten diagrams, Phys. Rev. Lett. 106 (2011) 091601 [arXiv: 1011.0780] [INSPIRE].

[33] S. Raju, Recursion relations for AdS/CFT correlators, Phys. Rev. D 83 (2011) 126002 [arXiv: 1102.4724] [INSPIRE]. 
[34] S. Albayrak, C. Chowdhury and S. Kharel, New relation for Witten diagrams, JHEP 10 (2019) 274 [arXiv: 1904.10043] [INSPIRE].

[35] S. Albayrak and S. Kharel, Towards the higher point holographic momentum space amplitudes, JHEP 02 (2019) 040 [arXiv:1810.12459] [INSPIRE].

[36] S. Albayrak, S. Kharel and D. Meltzer, On duality of color and kinematics in (A)dS momentum space, JHEP 03 (2021) 249 [arXiv:2012.10460] [INSPIRE].

[37] S. Albayrak, C. Chowdhury and S. Kharel, Study of momentum space scalar amplitudes in AdS spacetime, Phys. Rev. D 101 (2020) 124043 [arXiv:2001.06777] [InSPIRE].

[38] C. Armstrong, A.E. Lipstein and J. Mei, Color/kinematics duality in AdS $S_{4}$, JHEP 02 (2021) 194 [arXiv: 2012.02059] [INSPIRE].

[39] M.S. Costa, V. Gonçalves and J. Penedones, Spinning AdS propagators, JHEP 09 (2014) 064 [arXiv: 1404.5625] [INSPIRE].

[40] M.S. Costa, J. Penedones, D. Poland and S. Rychkov, Spinning conformal correlators, JHEP 11 (2011) 071 [arXiv: 1107.3554] [inSPIRE].

[41] O. Aharony, L.F. Alday, A. Bissi and E. Perlmutter, Loops in AdS from conformal field theory, JHEP 07 (2017) 036 [arXiv:1612.03891] [INSPIRE].

[42] J. Henriksson and T. Lukowski, Perturbative four-point functions from the analytic conformal bootstrap, JHEP 02 (2018) 123 [arXiv:1710.06242] [INSPIRE].

[43] L.F. Alday and A. Bissi, Loop corrections to supergravity on $A d S_{5} \times S^{5}$, Phys. Rev. Lett. 119 (2017) 171601 [arXiv:1706.02388] [INSPIRE].

[44] L.F. Alday, A. Bissi and E. Perlmutter, Holographic reconstruction of AdS exchanges from crossing symmetry, JHEP 08 (2017) 147 [arXiv:1705.02318] [INSPIRE].

[45] D. Mazac and M.F. Paulos, The analytic functional bootstrap. Part I. $1 D$ CFTs and $2 D$ S-matrices, JHEP 02 (2019) 162 [arXiv:1803.10233] [INSPIRE].

[46] D. Carmi, J. Penedones, J.A. Silva and A. Zhiboedov, Applications of dispersive sum rules:

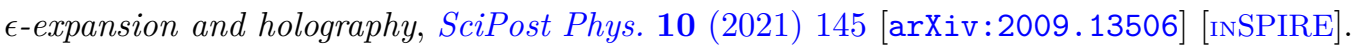

[47] S. Caron-Huot and A.-K. Trinh, All tree-level correlators in $A d S_{5} \times S_{5}$ supergravity: hidden ten-dimensional conformal symmetry, JHEP 01 (2019) 196 [arXiv:1809.09173] [INSPIRE].

[48] L.F. Alday and S. Caron-Huot, Gravitational S-matrix from CFT dispersion relations, JHEP 12 (2018) 017 [arXiv: 1711.02031] [INSPIRE].

[49] F. Aprile, J.M. Drummond, P. Heslop and H. Paul, Quantum gravity from conformal field theory, JHEP 01 (2018) 035 [arXiv:1706.02822] [INSPIRE].

[50] F. Aprile, J.M. Drummond, P. Heslop and H. Paul, Loop corrections for Kaluza-Klein AdS amplitudes, JHEP 05 (2018) 056 [arXiv:1711.03903] [INSPIRE].

[51] A. Bissi, G. Fardelli and A. Georgoudis, All loop structures in supergravity amplitudes on $A d S_{5} \times S^{5}$ from $C F T$, arXiv:2010.12557 [INSPIRE].

[52] A. Bissi, G. Fardelli and A. Georgoudis, Towards all loop supergravity amplitudes on $A d S_{5} \times S^{5}, \operatorname{arXiv}: 2002.04604$ [INSPIRE].

[53] P. Ferrero and C. Meneghelli, Bootstrapping the half-BPS line defect CFT in $N=4 S Y M$ at strong coupling, arXiv:2103.10440 [INSPIRE]. 
[54] P. Ferrero, K. Ghosh, A. Sinha and A. Zahed, Crossing symmetry, transcendentality and the Regge behaviour of $1 d$ CFTs, JHEP 07 (2020) 170 [arXiv: 1911.12388] [INSPIRE].

[55] D. Carmi, L. Di Pietro and S. Komatsu, A study of quantum field theories in AdS at finite coupling, JHEP 01 (2019) 200 [arXiv:1810.04185] [INSPIRE].

[56] A.L. Fitzpatrick, E. Katz, D. Poland and D. Simmons-Duffin, Effective conformal theory and the flat-space limit of AdS, JHEP 07 (2011) 023 [arXiv: 1007.2412] [INSPIRE].

[57] A.L. Fitzpatrick and J. Kaplan, Analyticity and the holographic S-matrix, JHEP 10 (2012) 127 [arXiv:1111.6972] [INSPIRE].

[58] I. Bertan and I. Sachs, Loops in anti-de Sitter space, Phys. Rev. Lett. 121 (2018) 101601 [arXiv: 1804.01880] [INSPIRE].

[59] I. Bertan, I. Sachs and E.D. Skvortsov, Quantum $\phi^{4}$ theory in AdS $S_{4}$ and its CFT dual, JHEP 02 (2019) 099 [arXiv: 1810.00907] [INSPIRE].

[60] M. Beccaria and A.A. Tseytlin, On boundary correlators in Liouville theory on AdS $S_{2}$, JHEP 07 (2019) 008 [arXiv: 1904.12753] [INSPIRE].

[61] A.L. Fitzpatrick and J. Kaplan, Unitarity and the holographic S-matrix, JHEP 10 (2012) 032 [arXiv: 1112.4845] [INSPIRE].

[62] D. Ponomarev, From bulk loops to boundary large-N expansion, JHEP 01 (2020) 154 [arXiv: 1908.03974] [INSPIRE].

[63] S. Giombi, C. Sleight and M. Taronna, Spinning AdS loop diagrams: two point functions, JHEP 06 (2018) 030 [arXiv:1708.08404] [INSPIRE].

[64] A. Costantino and S. Fichet, Opacity from loops in AdS, JHEP 02 (2021) 089 [arXiv:2011.06603] [INSPIRE].

[65] A. Antunes, M.S. Costa, T. Hansen, A. Salgarkar and S. Sarkar, The perturbative CFT optical theorem and high-energy string scattering in AdS at one loop, JHEP 04 (2021) 088 [arXiv: 2012.01515] [INSPIRE].

[66] D. Meltzer, E. Perlmutter and A. Sivaramakrishnan, Unitarity methods in AdS/CFT, JHEP 03 (2020) 061 [arXiv: 1912.09521] [INSPIRE].

[67] D. Meltzer and A. Sivaramakrishnan, CFT unitarity and the AdS Cutkosky rules, JHEP 11 (2020) 073 [arXiv: 2008.11730] [INSPIRE].

[68] S. Albayrak and S. Kharel, Spinning loop amplitudes in anti-de Sitter space, Phys. Rev. D 103 (2021) 026004 [arXiv:2006.12540] [INSPIRE].

[69] B. Nagaraj and D. Ponomarev, Spinor-helicity formalism for massless fields in AdS 4 . Part III. Contact four-point amplitudes, JHEP 08 (2020) 012 [arXiv: 2004.07989] [INSPIRE].

[70] X. Zhou, How to succeed at Witten diagram recursions without really trying, JHEP 08 (2020) 077 [arXiv : 2005.03031] [INSPIRE].

[71] L. Eberhardt, S. Komatsu and S. Mizera, Scattering equations in AdS: scalar correlators in arbitrary dimensions, JHEP 11 (2020) 158 [arXiv: 2007.06574] [INSPIRE].

[72] T. Kawano and K. Okuyama, Spinor exchange in AdS $S_{d+1}$, Nucl. Phys. B 565 (2000) 427 [hep-th/9905130] [INSPIRE].

[73] D.J. Gross and A. Neveu, Dynamical symmetry breaking in asymptotically free field theories, Phys. Rev. D 10 (1974) 3235 [inSPIRE]. 
[74] J. Zinn-Justin, Four fermion interaction near four-dimensions, Nucl. Phys. B 367 (1991) 105 [INSPIRE].

[75] B. Rosenstein, B. Warr and S.H. Park, Dynamical symmetry breaking in four Fermi interaction models, Phys. Rept. 205 (1991) 59 [INSPIRE].

[76] J. Liu, E. Perlmutter, V. Rosenhaus and D. Simmons-Duffin, d-dimensional SYK, AdS loops, and $6 j$ symbols, JHEP 03 (2019) 052 [arXiv:1808.00612] [INSPIRE].

[77] D. Carmi and S. Caron-Huot, A conformal dispersion relation: correlations from absorption, JHEP 09 (2020) 009 [arXiv:1910.12123] [INSPIRE].

[78] S. Caron-Huot, Analyticity in spin in conformal theories, JHEP 09 (2017) 078 [arXiv: 1703.00278] [INSPIRE].

[79] D. Mazac and M.F. Paulos, The analytic functional bootstrap. Part II. Natural bases for the crossing equation, JHEP 02 (2019) 163 [arXiv:1811.10646] [INSPIRE]. 